\title{
A Web Aggregation Approach for Distributed Randomized PageRank Algorithms*
}

\author{
Hideaki Ishii \\ Department of Computational Intelligence and Systems Science \\ Tokyo Institute of Technology \\ 4259 Nagatsuta-cho, Midori-ku, Yokohama 226-8502, Japan \\ E-mail: ishii@dis.titech.ac.jp
}

Roberto Tempo

CNR-IEIIT, Politecnico di Torino

Corso Duca degli Abruzzi 24, 10129 Torino, Italy

E-mail: roberto.tempo@polito.it

\author{
Er-Wei Bai \\ Department of Electrical and Computer Engineering, The University of Iowa \\ 4316 Seamans Center for the Engineering Arts and Sciences \\ Iowa City, IA 52242-1527, U.S.A. \\ and School of Electronics, Electrical Engineering and Computer Science \\ Queen's University, Belfast, BT7 1NN, U.K. \\ E-mail: er-wei-bai@uiowa.edu
}

September 29, 2018

\begin{abstract}
The PageRank algorithm employed at Google assigns a measure of importance to each web page for rankings in search results. In our recent papers, we have proposed a distributed randomized approach for this algorithm, where web pages are treated as agents computing their own PageRank by communicating with linked pages. This paper builds upon this approach to reduce the computation and communication loads for the algorithms. In particular, we develop a method to systematically aggregate the web pages into groups by exploiting the sparsity inherent in the web. For each group, an aggregated PageRank value is computed, which can then be distributed among the group members. We provide a distributed update scheme for the aggregated PageRank along with an analysis on its convergence properties. The method is especially motivated by results on singular perturbation techniques for large-scale Markov chains and multi-agent consensus.
\end{abstract}

${ }^{*}$ This paper has been accepted for publication in the IEEE Transactions on Automatic Control, 2012. This work was supported in part by the Ministry of Education, Culture, Sports, Science and Technology in Japan under Grant-in-Aid for Scientific Research, No. 21760323, the European Union Seventh Framework Programme [FP7/20072013] under grant agreement $n^{\circ} 257462$ HYCON2 Network of Excellence, and PRIN 2008 of Ministry of Education, Universities and Research of Italy. 


\section{Introduction}

When using the search engine Google, the rankings in search results take account of various aspects of web pages, but it has been acknowledged that the so-called PageRank algorithm provides crucial information for this purpose. This algorithm assigns to each web page a measure of its importance or popularity based solely on the link structure of the web. In particular, pages possessing more links, especially those from important pages, are given higher PageRank values, increasing the chance to be placed on the top of search results (see, e.g., $[9,11,37$ ).

One of the main challenges in implementing this algorithm is the size of the web. It is reported that the number of web page indices collected at Google is over 10 billion, causing serious issues for computation. Numerical methods for PageRank have been a subject of recent research. In the adaptive scheme of [33], computational resources are allocated to pages whose convergence to the PageRank values is slow. In [38], the problem size is reduced by treating the set of the socalled dangling nodes as a single node. The work of [5] employs techniques based on Monte Carlo simulation. On the other hand, numerical analysis methods known as asynchronous iterations [6] are applied to PageRank algorithms in [20,36]. In [41, a randomized algorithm is proposed based on stochastic descent methods with an explicit bound on the convergence rate. Moreover, the variations in PageRank values when the link structure changes have been studied from the viewpoint of fragile/uncertain links in [28] and also from optimization and linear algebra viewpoints in [19, 21, 1 .

In our recent paper [30], we focused on this algorithm and developed a distributed randomized approach for PageRank computation. From the control theoretic viewpoint, a key observation is that the PageRank computation shares several features with the multi-agent consensus problems, which have gained much attention in recent years; see, e.g., [1, 4, 6] and the references therein. Thus, we view the web as a network of agents having computation and communication capabilities. The idea is to let each web page, or the server that hosts it, compute its own PageRank value by communicating with neighboring pages connected by direct links. To realize asynchronous communication, it employs the so-called gossip protocol, where the pages randomly determine when information should be transmitted. Such a randomization-based method is motivated by the recent advances on probabilistic methods in systems and control [45] and has been adopted in the literature of multi-agent consensus (e.g., [8, 12, 13, 24, 26, 34, 44, 46, 47]). In [29, 31], we have also considered the effects of communication failures among the pages under this approach.

This paper aims at generalizing the distributed PageRank algorithms in [30] so that they can be used in an environment under limited resources. In particular, we develop efficient algorithms by reducing the amount of computation and communication loads. In such an environment, the computation of the true PageRank values may be difficult. Consequently, we provide an alternative method for finding a good approximate with bounds on the possible errors.

The proposed approach is based on a novel aggregation method of the original web to reduce

\footnotetext{
${ }^{1}$ In the area of e-commerce, various methods are known to enhance chances of specific web pages to be placed higher in search results, e.g., by including effective keywords in the pages. Such methods are sometimes referred to as search engine optimization (e.g., 14]). In these methods, the importance of PageRank is often emphasized through adding proper links.
} 
the size of the problem. The pages are first divided into a number of groups, for example, based on the hosts or the domains of the pages. It is known that most of the links in the web are intrahost ones [10,37], and thus the underlying graph has certain sparsity properties. To exploit such properties, we further aggregate the graph so that each group either (i) has more internal links than those going outside or (ii) consists of just one page. The aggregation procedure is easy to implement, employing a simple criterion, and can be applied to graphs with any link structures. Then, each group computes only one value in a decentralized manner via an enhanced version of the algorithms in [30]. This value represents the total value of the group members. Once this is computed, it can be distributed among the group members to determine their individual values. It is demonstrated theoretically and also through a numerical example that aggregation can reduce the computational cost while maintaining the accuracy and the convergence rate at a level similar to the non-aggregated full-order case.

The aggregation technique is particularly motivated by the singular perturbation analyses for large-scale systems in Markov chains [2,43] (see also [16,48]) and multi-agent consensus type problems [7, 15]. It is important to note that common in these works is the strong assumption on interactions among groups, requiring all groups to have only limited ratios of outgoing links towards other groups. This would not hold for the web graph where pages with many external links may not be common but indeed do exist. In the proposed aggregation method, such pages are treated as exceptions and are separated into groups of their own. We will later discuss the relation of our approach to those using singular perturbation techniques in more detail. Aggregation for PageRank computation has also been explored to obtain acceptable approximation in [37,49, by classical methods in the Markov chain literature. The paper [10] examines aggregation through extensive simulation using large data of the web. Recent works on aggregation for Markov chains can be found, e.g., in [23]. More generally, in the literature of complex networks, partitioning graphs into communities is a topic widely studied under various criteria for detecting communities; see, for example, [22] and the references therein.

From the viewpoint of distributed randomized approach, there are two new features in the current paper. First, the nodes initiate updates in a random manner as in the original algorithm of [30]. The difference is that an updating page transmits its value only to the pages to which it has outgoing links; this means that the extra data required in the previous algorithms on pages having incoming links towards this page becomes unnecessary. Second, each node can further divide its linked pages into several groups and communicates with them separately under possibly different update probabilities. These features are useful to reduce the amount of the overall communication, especially for pages with many links.

This paper is organized as follows: We first give a brief overview of the PageRank problem in Section 2, In Section 3, we introduce the approach for web aggregation and the communication protocol among agents and then formulate the problem of computing the PageRank values via web aggregation. In Section 4 , the main results on the aggregation-based algorithm are presented along with an analysis on error bounds. Discussions on the relation to singular perturbation techniques are given in Section 5, In Section 6, the distributed randomized approach is developed for the part 
of the proposed algorithm based on the reduced-order recursion. We provide a numerical example in Section 7 to illustrate the results. The paper is finally concluded in Section 8 , Parts of the results in this paper have appeared in preliminary forms in [31,32].

Notation: For vectors and matrices, inequalities are used to denote entry-wise inequalities: For $X, Y \in \mathbb{R}^{n \times m}, X \leq Y$ implies $x_{i j} \leq y_{i j}$ for $i=1, \ldots, n$ and $j=1, \ldots, m$; in particular, we say that the matrix $X$ is nonnegative if $X \geq 0$ and positive if $X>0$. A probability vector is a nonnegative vector $v \in \mathbb{R}^{n}$ such that $\sum_{i=1}^{n} v_{i}=1$. A matrix $X \in \mathbb{R}^{n \times n}$ is said to be (column) stochastic if it is nonnegative and each column sum equals 1 , i.e., $\sum_{i=1}^{n} x_{i j}=1$ for each $j$. Let $\mathbf{1}_{n} \in \mathbb{R}^{n}$ be the vector whose entries are all 1 as $\mathbf{1}_{n}:=\left[\begin{array}{lll}1 & \cdots & 1\end{array}\right]^{T}$. Similarly, $S \in \mathbb{R}^{n \times n}$ is the matrix with all entries being 1 . The spectral radius of the matrix $X \in \mathbb{R}^{n \times n}$ is denoted by $\rho(X)$.

\section{The PageRank problem}

In this section, the PageRank problem is briefly described based on, e.g., [9,11,37].

Consider the directed graph $\mathcal{G}=(\mathcal{V}, \mathcal{E})$ representing a network of $n$ web pages. Here, $\mathcal{V}:=$ $\{1,2, \ldots, n\}$ is the set of nodes corresponding to the web page indices while $\mathcal{E} \subset \mathcal{V} \times \mathcal{V}$ is the set of edges for the links among the pages. The node $i$ is connected to the node $j$ by an edge, i.e., $(i, j) \in \mathcal{E}$, if page $i$ has an outgoing link to page $j$.

The objective of the PageRank algorithm is to assign some measure of importance to each web page. The PageRank value of page $i \in \mathcal{V}$ is given by $x_{i}^{*} \in[0,1]$. The relation $x_{i}^{*}>x_{j}^{*}$ implies that page $i$ has higher rank than page $j$. The pages are ranked according to the rule that a page having more links, especially those from important pages, becomes more important. This is done in such a way that the value of one page equals the sum of the contributions from all pages that have links to it. Let the values be in the vector form as $x^{*} \in[0,1]^{n}$. Then, the PageRank vector $x^{*}$ is defined by

$$
x^{*}=A x^{*}, \quad x^{*} \in[0,1]^{n}, \quad \mathbf{1}_{n}^{T} x^{*}=1,
$$

where the link matrix $A=\left(a_{i j}\right) \in \mathbb{R}^{n \times n}$ is given by $a_{i j}=1 / n_{j}$ if $(j, i) \in \mathcal{E}$ and 0 otherwise, and $n_{j}$ is the number of outgoing links of page $j$. Hence, the value vector $x^{*}$ is a nonnegative unit eigenvector corresponding to the eigenvalue 1 of $A$.

In general, for this eigenvector to exist and then to be unique, it is sufficient that the web as a graph is strongly connected [27]2. However, the web is known not to be strongly connected. Thus, the convention is to slightly modify the problem as follows. First, to simplify the discussion, we redefine the graph, and thus the matrix $A$, by bringing in artificial links for nodes with no outgoing links such as PDF files. This can be done by adding links back to the pages having links to such pages. As a result, the link matrix $A$ becomes a stochastic matrix, that is, $\sum_{i=1}^{n} a_{i j}=1$ for each $j$. This implies that there exists at least one eigenvalue equal to 1 . To guarantee the uniqueness of this eigenvalue, let $m$ be a parameter such that $m \in(0,1)$, and let the modified link matrix $M \in \mathbb{R}^{n \times n}$ be defined by $M:=(1-m) A+(m / n) S$, where $S \in \mathbb{R}^{n \times n}$ is the matrix whose entries are all 1 .

\footnotetext{
${ }^{2} \mathrm{~A}$ directed graph is said to be strongly connected if for any two nodes $i, j \in \mathcal{V}$, there exists a sequence of edges which connects node $i$ to node $j$.
} 
Notice that $M$ is a positive stochastic matrix 3. By Perron's theorem [27], the eigenvalue 1 is of multiplicity 1 and is the unique eigenvalue with maximum magnitude. Further, the corresponding eigenvector is positive. Hence, we redefine the value vector $x^{*}$ by using $M$ as follows.

Definition 2.1 The PageRank value vector $x^{*}$ is given by

$$
x^{*}=M x^{*}, \quad x^{*} \in[0,1]^{n}, \quad \mathbf{1}_{n}^{T} x^{*}=1 .
$$

Due to the large dimension of the link matrix $M$, the computation of $x^{*}$ is difficult. The solution employed in practice is based on the power method given by the recursion

$$
x(k+1)=M x(k)=(1-m) A x(k)+\frac{m}{n} \mathbf{1}_{n},
$$

where $x(k) \in \mathbb{R}^{n}$ and the initial vector $x(0) \in \mathbb{R}^{n}$ is a probability vector. The second equality above follows from the fact $S x(k)=\mathbf{1}_{n}, k \in \mathbb{Z}_{+}$. For implementation, the form on the far right-hand side is important, using only the sparse matrix $A$ and not the dense matrix $M$. This method asymptotically finds the value vector as shown below [27].

Lemma 2.2 In the update scheme (3), for any initial state $x(0)$ that is a probability vector, it holds that $x(k) \rightarrow x^{*}$ as $k \rightarrow \infty$.

\section{Problem formulation}

In this section, we introduce the problem setting for the distributed computation of the aggregated PageRank. Following the randomized distributed approach proposed in [30], we view the web as a network of agents having computation and communication capabilities. The focus here is to extend the distributed algorithm of [30] so that it can be executed with reduced computation and communication to determine approximate values of the exact PageRank. In what follows, we present the procedure for aggregation of the web and then introduce the communication protocol.

\subsection{Web aggregation}

In the proposed approach, the original web is aggregated by assigning each page into a number of groups and then each group computes one value, which is the sum of the values of the group members. We aggregate the pages sharing the following three properties: (i) The pages are placed under the same host/server so that their values can be computed together. (ii) Each group has a sufficiently large number of internal links. More specifically, pages have more links within their own groups than those pointing at pages that belong to other groups having multiple members. (iii) Group members are expected to take similar values in PageRank; this may be known from past computations and/or the link structure. We will show that the process of grouping can be done locally at each host.

\footnotetext{
${ }^{3}$ In the original algorithm in [9], a typical value for $m$ is reported to be $m=0.15$, but no specific reason is given for this choice. We will use this value throughout this paper.
} 
We develop a novel aggregation approach by exploiting sparsity properties that the web inherently has, as stated by (ii) above. The particular approach has a close relation to the singular perturbation analysis for large-scale systems with network structures [2, 7, 15, 43]. The method there however requires a stronger sparsity notion on the underlying graph, which seems difficult to expect in the web. Hence, necessary modifications will be made in the approach. The relation among these papers will be discussed in Section 5 .

First, partition the original web graph $\mathcal{G}=(\mathcal{V}, \mathcal{E})$ and construct the aggregated graph denoted by $\widetilde{\mathcal{G}}=(\widetilde{\mathcal{V}}, \widetilde{\mathcal{E}})$ as follows:

(i) The node set is given by $\widetilde{\mathcal{V}}:=\{1,2, \ldots, r\}$, and each node $i$ represents a partition set $\mathcal{U}_{i}$ of $\mathcal{V}$, that is, $\bigcup_{i} \mathcal{U}_{i}=\mathcal{V}$ and $\mathcal{U}_{i} \cap \mathcal{U}_{j}=\emptyset, \forall i \neq j$. We call the set $\mathcal{U}_{i}$ a group of pages. Let $r$ be the number of groups, and let $\widetilde{n}_{i}$ be the number of pages in group $\mathcal{U}_{i}$. Thus, $\sum_{i=1}^{r} \widetilde{n}_{i}=n$.

(ii) The edge set $\widetilde{\mathcal{E}}=\widetilde{\mathcal{V}} \times \widetilde{\mathcal{V}}$ satisfies that if $\left(i_{1}, i_{2}\right) \in \mathcal{E}$, then $\left(h\left(i_{1}\right), h\left(i_{2}\right)\right) \in \widetilde{\mathcal{E}}$, where $h: \mathcal{V} \rightarrow \widetilde{\mathcal{V}}$ is the function indicating the group $j$ that the web page $i$ belongs to such that $h(i)=j$, or $i \in \mathcal{U}_{j}$.

To simplify the notation, without loss of generality, we assume that in the PageRank vector $x^{*}$, the first $\widetilde{n}_{1}$ entries correspond to the pages belonging to group $\mathcal{U}_{1}$, and the following $\widetilde{n}_{2}$ entries are for those in group $\mathcal{U}_{2}$, and so on.

We make the following assumption regarding the grouping. It says that each group should have a sufficiently small number of external links compared to internal ones. Recall from (1) that $n_{i}$ denotes the number of outgoing links of page $i$, and let $n_{\text {ext }, i}$ be the number of outgoing links from page $i$ to groups having more than one page. Following [15], we define the node parameter $\delta_{i}$ of page $i$ by

$$
\delta_{i}:=\frac{n_{\mathrm{ext}, i}}{n_{i}}, \quad i=1, \ldots, n .
$$

Assumption 3.1 Given the bound $\delta \in(0,1)$ on node parameters, each group $j$ satisfies one of the following conditions:

(i) For each page $i$ in group $j$, it holds that $\delta_{i} \leq \delta$.

(ii) Group $j$ consists of only one page.

In view of (ii) above, groups with one member are called single groups; denote by $r_{1}$ the number of such groups. These groups represent exceptional pages having high ratios of external links.

After the groups of pages are determined satisfying the assumptions above, we consider the values that represent the groups. For this purpose, in the update scheme, we employ the coordinate transformation $\widetilde{x}(k):=V x(k)$ via the matrix $V=\left[V_{1}^{T} V_{2}^{T}\right]^{T} \in \mathbb{R}^{n \times n}$ given by

$$
\begin{aligned}
V_{1} & :=\operatorname{bdiag}\left(\mathbf{1}_{\widetilde{n}_{i}}^{T}\right) \in \mathbb{R}^{r \times n}, \\
V_{2} & :=\operatorname{bdiag}\left(\left[I_{\widetilde{n}_{i}-1} 0\right]-\frac{1}{\widetilde{n}_{i}} \mathbf{1}_{\widetilde{n}_{i}-1} \mathbf{1}_{\widetilde{n}_{i}}^{T}\right) \in \mathbb{R}^{(n-r) \times n},
\end{aligned}
$$


where bdiag $\left(X_{i}\right)$ denotes a block-diagonal matrix whose $i$ th diagonal block is $X_{i}$. It should be noted that $V_{1}$ and $V_{2}$ are block-diagonal matrices containing $r$ and $r-r_{1}$ blocks, respectively. They have simple structures, depending only on the sizes $\widetilde{n}_{i}$ of the groups. Note that in $V_{2}$, if the $i$ th group is a single one (i.e., $\widetilde{n}_{i}=1$ ), then the $i$ th block has the size $0 \times 1$, meaning that the corresponding column is zero. Moreover, $V_{1}$ and $V_{2}$ are orthogonal: $V_{1} V_{2}^{T}=0$.

The PageRank vector $\widetilde{x}^{*}$ and the state $\widetilde{x}(k)$ after the transformation are partitioned as

$$
\widetilde{x}^{*}=\left[\begin{array}{c}
\widetilde{x}_{1}^{*} \\
\widetilde{x}_{2}^{*}
\end{array}\right]:=\left[\begin{array}{c}
V_{1} \\
V_{2}
\end{array}\right] x^{*}, \quad \widetilde{x}(k)=\left[\begin{array}{l}
\widetilde{x}_{1}(k) \\
\widetilde{x}_{2}(k)
\end{array}\right]:=\left[\begin{array}{l}
V_{1} \\
V_{2}
\end{array}\right] x(k) .
$$

In the first part $\widetilde{x}_{1}^{*}$ of the PageRank vector in the new coordinate, the $i$ th entry is the total value of the members in group $i$; this vector $\widetilde{x}_{1}^{*}$ is referred to as the aggregated PageRank. In the second part $\widetilde{x}_{2}^{*}$, each entry represents the difference between a page value and the average value of the group members. In the distributed algorithm developed in Section 6, the objective is to compute the aggregated PageRank $\widetilde{x}_{1}^{*}$ in a recursive fashion via information exchange only among groups. After this is completed, the second part $\widetilde{x}_{2}^{*}$ should be obtained. It will be shown that in this stage, transmissions among pages in different groups is necessary, but only once during the execution of the algorithm. Hence, reduced communication load can be expected in particular when $r$ is small.

Remark 3.2 For a given bound $\delta$ on the node parameters, a simple grouping procedure for Assumption 3.1 to hold can be described as follows. The pages are initially grouped based on their hosts, so the computation of the node parameters $\delta_{i}$ in (4) can be done locally. Any page $i$ whose $\delta_{i}$ does not satisfy the condition (i) is taken out from the group; such pages are treated as single groups, for which the condition (ii) applies. Other pages still belong to the same group, and thus their parameters $\delta_{i}$ are updated and then checked whether (i) holds for this new group. These steps are repeated until all pages under one host satisfy the assumption. It is clear that for any given bound $\delta$ on the node parameters, this procedure will terminate. It should be noted that there is a tradeoff between the parameter $\delta$ and the number $r$ of groups: Smaller $\delta$ implies larger $r$, and vice versa.

\subsection{Communication protocol via random gossipping}

We next discuss the communication among groups in the proposed distributed algorithm.

For the computation of $\widetilde{x}_{1}(k)$, the groups send their values to linked groups. Here, we employ a gossip-type protocol, where the groups decide to communicate with their linked neighbors at random times. Such a protocol is based on local information only and does not require a common clock. It is thus useful in realizing asynchronous algorithms for a network of agents (e.g., [8,12,13, 24, 26, 34, 44, 46, 47]).

In the aggregated graph $\widetilde{\mathcal{G}}=(\widetilde{\mathcal{V}}, \widetilde{\mathcal{E}})$, the nodes exchange their values over their outgoing links. Denote by $\widetilde{\mathcal{V}}_{i}$ the set of indices of the groups having links from node $i$ as

$$
\widetilde{\mathcal{V}}_{i}:=\{j \in \widetilde{\mathcal{V}}:(i, j) \in \widetilde{\mathcal{E}}, j \neq i\} .
$$


Here, we allow node $i$ to communicate with a subset of $\widetilde{\mathcal{V}}_{i}$ at a time. This helps to reduce the instantaneous communication load especially for nodes having many links. For this purpose, we partition $\widetilde{\mathcal{V}}_{i}$ into the sets $\widetilde{\mathcal{V}}_{i, 1}, \ldots, \widetilde{\mathcal{V}}_{i, g_{i}}$, where $g_{i}$ is the number of partition sets, i.e., it holds that

$$
\widetilde{\mathcal{V}}_{i}=\bigcup_{\ell=1}^{g_{i}} \widetilde{\mathcal{V}}_{i, \ell}, \quad \widetilde{\mathcal{V}}_{i, \ell} \cap \widetilde{\mathcal{V}}_{i, j}=\emptyset, \quad \forall \ell \neq j
$$

For each node $i \in \widetilde{\mathcal{V}}$, let $\eta_{i}(k) \in\left\{0,1, \ldots, g_{i}\right\}$ be the i.i.d. random process that specifies the set of nodes to which it sends the value $\left(\widetilde{x}_{1}(k)\right)_{i}$ at time $k$. That is,

$$
\eta_{i}(k)= \begin{cases}\ell & \text { if node } i \text { sends its value to nodes in } \tilde{\mathcal{V}}_{i, \ell}, \ell=1,2, \ldots, g_{i}, \\ 0 & \text { if node } i \text { does not communicate }\end{cases}
$$

for $k \in \mathbb{Z}_{+}$. The probability distribution of this process is given as

$$
\alpha_{i, \ell}=\operatorname{Prob}\left\{\eta_{i}(k)=\ell\right\}, \quad \ell=0,1, \ldots, g_{i}, \quad k \in \mathbb{Z}_{+} .
$$

The update probabilities $\alpha_{i, \ell} \in(0,1)$ are chosen so as to satisfy the condition

$$
\sum_{\ell=0}^{g_{i}} \alpha_{i, \ell}=1, \quad i \in \widetilde{\mathcal{V}}
$$

The main problem studied in this paper can be roughly restated as follows: Design a distributed randomized algorithm for computing approximated PageRank values such that (i) the groups compute $\widetilde{x}_{1}(k)$, the total values of their member pages, following the gossip protocol for communication and then (ii) from $\widetilde{x}_{1}(k)$, the PageRank vector $x(k)$ and, in particular, the values for individual pages are obtained.

We characterize the web aggregation approach in Section 4 along with error analyses for the aggregated PageRank. Then, in Section 6, the distributed randomized algorithm of reduced order for computing the group values $\widetilde{x}_{1}(k)$ is discussed.

\section{Aggregation-based PageRank computation}

In this section, we present the approach for aggregating the web graph and then propose an approximated version of the PageRank that can be computed from a lower-order update scheme.

\subsection{Definition of aggregated PageRank}

We begin by analyzing the centralized update scheme of (3) described in Section 2 when the state is transformed as $\widetilde{x}(k)=V x(k)$ by ([6) . Let $\widetilde{A}:=V A V^{-1}$ be the link matrix in the new coordinate. Partition it in accordance with the dimensions of $\widetilde{x}_{1}(k)$ and $\widetilde{x}_{2}(k)$ as

$$
\widetilde{A}=\left[\begin{array}{ll}
\widetilde{A}_{11} & \widetilde{A}_{12} \\
\widetilde{A}_{21} & \widetilde{A}_{22}
\end{array}\right]
$$


with $\widetilde{A}_{11} \in \mathbb{R}^{r \times r}$. The update scheme in (3) can be expressed as

$$
\begin{aligned}
& \widetilde{x}_{1}(k+1)=(1-m) \widetilde{A}_{11} \widetilde{x}_{1}(k)+(1-m) \widetilde{A}_{12} \widetilde{x}_{2}(k)+\frac{m}{n} u, \\
& \widetilde{x}_{2}(k+1)=(1-m) \widetilde{A}_{21} \widetilde{x}_{1}(k)+(1-m) \widetilde{A}_{22} \widetilde{x}_{2}(k),
\end{aligned}
$$

where $u:=V_{1} \mathbf{1}_{n}=\left[\widetilde{n}_{1} \cdots \widetilde{n}_{r}\right]^{T}$; we also used the fact $V_{2} \mathbf{1}_{n}=0$. The initial states are such that $\widetilde{x}_{1}(0) \geq 0$ and $\mathbf{1}_{r}^{T} \widetilde{x}_{1}(0)=1$. The steady state of this scheme is the transformed PageRank vector $\widetilde{x}^{*}$ given in (6).

Now, to derive an approximated version of the update scheme above, we focus on the characteristics of the submatrices $\widetilde{A}_{i j}$. The transformation matrix $V$ in (5) has a simple structure, and the advantage is that its inverse can be found in an explicit form, which will be useful in our analysis. Denote the inverse by $W:=V^{-1}$. It can be partitioned as $W=\left[W_{1} W_{2}\right]$ where

$$
\begin{aligned}
W_{1} & :=\operatorname{bdiag}\left(\frac{1}{\widetilde{n}_{i}} \mathbf{1}_{\widetilde{n}_{i}}\right) \in \mathbb{R}^{n \times r}, \\
W_{2} & :=\operatorname{bdiag}\left(\left[\begin{array}{c}
I_{\widetilde{n}_{i}-1} \\
-\mathbf{1}_{\widetilde{n}_{i}-1}^{T}
\end{array}\right]\right) \in \mathbb{R}^{n \times(n-r)} .
\end{aligned}
$$

Again, $W_{1}$ and $W_{2}$ are block-diagonal matrices with $r$ and $r-r_{1}$ blocks, respectively. Moreover, the rows in $W_{2}$ that correspond to single groups are zero. It is obvious that $V_{1} W_{1}=I, V_{1} W_{2}=0$, $V_{2} W_{1}=0$, and $V_{2} W_{2}=I$.

Based on the approach studied in [43, the key observation in the proposed aggregation is that the matrix $A$ can be decomposed into three parts as

$$
A=I+A_{\text {int }}+A_{\text {ext }} .
$$

Here, the internal link matrix $A_{\text {int }}$ is block diagonal; its $i$ th block is of the size $\widetilde{n}_{i} \times \widetilde{n}_{i}$, whose nondiagonal entries are the same as those of $A$, but its diagonal entries are chosen so that the column sums are zero. This implies that $I+A_{\text {int }}$ is a block-diagonal stochastic matrix. Hence, it easily follows that

$$
V_{1} A_{\text {int }}=0
$$

On the other hand, the external link matrix $A_{\text {ext }}$ contains all elements in $A$ which are not in the block-diagonal $A_{\text {int }}$ while its diagonal entries are chosen so that each column sum equals zero. Let $A_{\text {ext0 }}$ be an $n \times n$ matrix whose $j$ th column is the same as that of $A_{\text {ext }}$ if page $j$ belongs to a non-single group (i.e., with more than one member) and zero otherwise for $j=1, \ldots, n$. By the definition of $W_{2}$, it is simple to check that

$$
A_{\text {ext }} W_{2}=A_{\text {ext } 0} W_{2} .
$$

By using the facts $W=V^{-1}$, (13) , (14), and (15), the submatrices of $\widetilde{A}$ in (10) can be expressed as

$$
\left[\begin{array}{cc}
\widetilde{A}_{11} & \widetilde{A}_{12} \\
\widetilde{A}_{21} & \widetilde{A}_{22}
\end{array}\right]=\left[\begin{array}{ll}
V_{1} A W_{1} & V_{1} A W_{2} \\
V_{2} A W_{1} & V_{2} A W_{2}
\end{array}\right]=\left[\begin{array}{cc}
I+V_{1} A_{\text {ext }} W_{1} & V_{1} A_{\text {ext } 0} W_{2} \\
V_{2}\left(A_{\text {int }}+A_{\text {ext }}\right) W_{1} & I+V_{2}\left(A_{\text {int }}+A_{\text {ext0 }}\right) W_{2}
\end{array}\right] .
$$


For later use, from $\widetilde{A}_{22}$, we remove $A_{\text {ext0 }}$ to obtain the block-diagonal matrix $\widetilde{A}_{22}^{\prime}$ given by

$$
\widetilde{A}_{22}^{\prime}:=I+V_{2} A_{\text {int }} W_{2} .
$$

The following results are helpful to justify the approach, as we shall see later.

Lemma $4.1 \quad$ (i) The matrix $\widetilde{A}_{11}$ is stochastic.

(ii) The matrix $\widetilde{A}_{22}^{\prime}$ in (17) has spectral radius smaller than or equal to 1 .

(iii) Under Assumption 3.1, it holds that $\left\|A_{\text {ext0 }}\right\|_{1} \leq 2 \delta$.

Proof: (i) It is clear that $\widetilde{A}_{11}$ is nonnegative and satisfies $\mathbf{1}_{r}^{T} \widetilde{A}_{11}=\mathbf{1}_{r}^{T} V_{1} A W_{1}=\mathbf{1}_{r}^{T}$.

(ii) The matrix $I+A_{\text {int }}$ is stochastic. In particular, it has $r$ diagonal blocks, which are all stochastic. Thus, $I+A_{\text {int }}$ has at least $r$ eigenvalues equal to 1 , and the rest have magnitude less than or equal to 1 . The transformation matrix $V$ is composed of orthogonal rows, and thus $\operatorname{Im} V_{2}^{T}=$ $\left(\operatorname{Im} V_{1}^{T}\right)^{\perp}$. However, $\operatorname{Im} V_{1}^{T}$ is the left eigenspace of $I+A_{\text {int }}$ corresponding to $r$ of the eigenvalues 1 . This implies that $\operatorname{Im} V_{2}^{T}$ spans the eigenspace for the rest of the eigenvalues of $I+A_{\text {int }}$; let $\lambda$ be any such eigenvalue. Then, there exists a vector $v \in \mathbb{C}^{n-r}$ such that $v^{T} V_{2}\left(I+A_{\text {int }}\right)=\lambda v^{T} V_{2}$. Notice $V_{2} W_{2}=I$, and thus $v^{T} V_{2}\left(I+A_{\text {int }}\right) W_{2}=\lambda v^{T}$, showing that $\lambda$ is an eigenvalue of $V_{2}\left(I+A_{\text {int }}\right) W_{2}$ as well. Therefore, we conclude that $\rho\left(\widetilde{A}_{22}^{\prime}\right)=\rho\left(V_{2}\left(I+A_{\text {int }}\right) W_{2}\right) \leq 1$.

(iii) For $i \neq j$, the $(i, j)$ entry of $A_{\text {ext0 }}$ is nonzero (and equals $a_{i j}=1 / n_{j}$ ) if and only if page $j$ has a link to page $i$, and moreover pages $i$ and $j$ belong to different groups, each of which having multiple group members. By assumption, for each column, the sum of its off-diagonal entries is less than or equal to $\delta$, but each column sum equals zero. Hence, the 1-norm of $A_{\text {exto }}$ is bounded by $2 \delta$.

An important implication of (ii) and (iii) of this lemma is that if the node parameter $\delta$ in Assumption 3.1 is sufficiently small, then the matrix $(1-m) \widetilde{A}_{22}$ is stables; this is because from (16), we have $\widetilde{A}_{22}=I+V_{2}\left(A_{\text {int }}+A_{\text {ext } 0}\right) W_{2}=\widetilde{A}_{22}^{\prime}+V_{2} A_{\text {ext } 0} W_{2}$, where $A_{\text {ext0 }}$ is proportional to $\delta$; recall also that $\widetilde{A}_{22}^{\prime}$ is a block-diagonal matrix, which will become crucial from the computational viewpoint. We now come to the idea of how to approximate the scheme (11) and (12). First, express (12) for $\widetilde{x}_{2}(k)$ using its steady state (i.e., $\left.\widetilde{x}_{2}(k+1)=\widetilde{x}_{2}(k)\right)$ as

$$
\widetilde{x}_{2}(k)=(1-m)\left[I-(1-m) \widetilde{A}_{22}\right]^{-1} \widetilde{A}_{21} \widetilde{x}_{1}(k),
$$

where the matrix $I-(1-m) \widetilde{A}_{22}$ is nonsingular. This expression is motivated by the time-scale separation in singular perturbation based approaches. Substituting this into the recursion (11) for $\widetilde{x}_{1}(k)$ yields

$$
\widetilde{x}_{1}(k+1)=(1-m)\left\{\widetilde{A}_{11}+(1-m) \widetilde{A}_{12}\left[I-(1-m) \widetilde{A}_{22}\right]^{-1} \widetilde{A}_{21}\right\} \widetilde{x}_{1}(k)+\frac{m}{n} u .
$$

Note that if this recursion is stable, then the steady states of the scheme above with (18) and (19) become the same as those of (11) and (12); they are equal to the transformed PageRank $\widetilde{x}^{*}$ in (6).

\footnotetext{
${ }^{4} \mathrm{~A}$ matrix is said to be stable if it is Schur stable, that is, if all of its eigenvalues have magnitude less than 1.
} 
In this approximate form (18) and (19), the scheme requires the recursive computation of only the first state $\widetilde{x}_{1}(k)$, whose dimension equals the number $r$ of groups. It thus appears that information should be exchanged only among groups. However, we notice that the term $\widetilde{A}_{12}[I-$ $\left.(1-m) \widetilde{A}_{22}\right]^{-1} \widetilde{A}_{21} \widetilde{x}_{1}(k)$ involves the product of vectors of dimension $n-r$ and consequently may not be suitable for distributed computation.

To reduce the computation and communication, we further simplify the scheme by relaxing the objective to that of computing the approximated version of the state $\widetilde{x}(k)$. Specifically, we modify the scheme (18) and (19) above under the assumption that $\delta$ is sufficiently small. The scheme consists of three steps and is given as follows.

Algorithm 4.2 1. Take the initial state $\widetilde{x}_{1}(0) \in \mathbb{R}^{r}$ as a probability vector. At each time $k$, compute the first state $\widetilde{x}_{1}(k) \in \mathbb{R}^{r}$ via the reduced-order recursion

$$
\widetilde{x}_{1}(k+1)=(1-m) \widetilde{A}_{11} \widetilde{x}_{1}(k)+\frac{m}{n} u .
$$

2. After the updates for $\widetilde{x}_{1}(k)$ converge, compute the second state $\widetilde{x}_{2}(k) \in \mathbb{R}^{n-r}$ by

$$
\widetilde{x}_{2}(k)=(1-m)\left[I-(1-m) \widetilde{A}_{22}^{\prime}\right]^{-1} \widetilde{A}_{21} \widetilde{x}_{1}(k) .
$$

3. The state is transformed back in the original coordinate by

$$
x(k)=W \widetilde{x}(k)=W_{1} \widetilde{x}_{1}(k)+W_{2} \widetilde{x}_{2}(k) .
$$

In summary, we obtained Algorithm 4.2, which is an approximated version of the the scheme in (11) and (12). The approach in the derivation outlined above is (i) to use the steady state of $\widetilde{x}_{2}(k)$, and then (ii) to assume small $\delta$ so that $\widetilde{A}_{12}$ and the entries of $\widetilde{A}_{22}$ outside the diagonal blocks become small (due to Lemma 4.1(iii)). In particular, the original scheme is triagonalized by replacing $\widetilde{A}_{12}$ with zeros; as a result, in the first step of the algorithm, only the $r$-dimensional dynamics for the group values remains. Moreover, in the second step, $\widetilde{A}_{22}^{\prime}$ is block diagonal, so the matrix inversion in (21) can be done at each group (while the first step is running). The level of approximation is guaranteed via detailed analyses provided in Theorems 4.5 and 4.7 in the next subsection.

The convergence of this scheme is outlined below. Similarly to Definition 2.1 for the original PageRank vector $x^{*}$, let $\widetilde{x}_{1}^{\prime} \in \mathbb{R}^{r}$ be the eigenvector of the matrix $(1-m) \widetilde{A}_{11}+(m / n) u \mathbf{1}_{r}^{T}$ corresponding to eigenvalue 1 as

$$
\widetilde{x}_{1}^{\prime}=\left[(1-m) \widetilde{A}_{11}+\frac{m}{n} u \mathbf{1}_{r}^{T}\right] \widetilde{x}_{1}^{\prime}, \quad \widetilde{x}_{1}^{\prime} \in[0,1]^{r}, \quad \mathbf{1}_{r}^{T} \widetilde{x}_{1}^{\prime}=1 .
$$

This eigenvector exists and is unique because $\widetilde{A}_{11}$ is stochastic by Lemma 4.1(i) and moreover, $u / n$ is a positive probability vector by definition; hence, this matrix $(1-m) \widetilde{A}_{11}+(m / n) u \mathbf{1}_{r}^{T}$ is positive stochastic and Perron's theorem [27] can be applied. Then, let

$$
\widetilde{x}^{\prime}:=\left[\begin{array}{l}
\widetilde{x}_{1}^{\prime} \\
\widetilde{x}_{2}^{\prime}
\end{array}\right], \text { where } \widetilde{x}_{2}^{\prime}:=(1-m)\left[I-(1-m) \widetilde{A}_{22}^{\prime}\right]^{-1} \widetilde{A}_{21} \widetilde{x}_{1}^{\prime}
$$


Table 1: Comparison of operation costs with communication among groups

\begin{tabular}{lll}
\hline Algorithm & Equation & Bound on numbers of operations \\
\hline Original & $(\underline{3})$ & $O\left(\left(2 f_{0}(A)+n\right) \bar{k}_{1}\right)$ \\
Aggregation based & $(20)$ & $O\left(\left(2 f_{0}\left(\widetilde{A}_{11}\right)+r\right) \bar{k}_{2}+f_{0}\left(A_{\text {ext }}\right)+n+r\right)$ \\
& $(21)$ & $O\left(2 f_{0}(A)+2 n+r\right)$ \\
\hline
\end{tabular}

$f_{0}(\cdot)$ : The number of nonzero entries of a matrix $\bar{k}_{1}, \bar{k}_{2}$ : The numbers of steps in the recursions

The first part $\widetilde{x}_{1}^{\prime}$ is the approximate of the aggregated PageRank $\widetilde{x}_{1}^{*}$; with some abuse of terminology, it will also be called the aggregated PageRank. Finally, we transform this $\widetilde{x}^{\prime}$ back to the original coordinate, and let

$$
x^{\prime}:=V^{-1} \widetilde{x}^{\prime} .
$$

The update scheme in the algorithm is guaranteed to converge to this approximated PageRank vector $x^{\prime}$. We state this fact as a proposition, which follows from Lemma 2.2.

Proposition 4.3 In the three-step update scheme in (201)-(22), for any initial vector $\widetilde{x}_{1}(0)$ that is a probability vector, it holds that the state $x(k)$ converges to $x^{\prime}$ in (25) as $k \rightarrow \infty$.

We have a few remarks regarding this algorithm from the viewpoint of distributed computation. In the first step (20), the $r$-dimensional state $\widetilde{x}_{1}(k)$ represents the group values. This step requires exchange of states only among groups and not among individual pages. Hence, it is suitable for distributed computation. Once it reaches the steady state, the other two steps should be carried out. The second step (21) requires transmission over most links in the web for communicating the $(n-r)$-dimensional vector $\widetilde{A}_{21} \widetilde{x}_{1}(k)$. Nevertheless, the subsequent computation in this step as well as the third step (22) can be done locally within each group. This is because the matrices $I-(1-m) \widetilde{A}_{22}^{\prime}, W_{1}$, and $W_{2}$ are all block diagonal.

Remark 4.4 The computational advantage of the aggregation-based approach can be highlighted in terms of its operation cost [25,38]. Table1 summarizes the numbers of operations for the original scheme (3) and the proposed scheme (20) $-(22)$ in Algorithm 4.2. Here, $f_{0}(A)$ denotes the number of nonzero entries in the link matrix $A$. For a sparse matrix, its product with a vector requires operations of order $2 f_{0}(A)$. Also, $\bar{k}_{1}$ and $\bar{k}_{1}$ are the numbers of steps required for the convergence of the recursions; for termination criteria, see, e.g., [33] for the centralized case and [30] for the distributed case.

For the proposed scheme, the operations that involve interaction among groups via communication are shown; other steps can be done decentrally and are of polynomial orders of $n_{i}$ for group $i$. The first step (20) requires the computation of $\widetilde{A}_{11}=I+V_{1} A_{\mathrm{ext}} W_{1}$ and the iteration. For the second step (21), we counted the multiplication of $\widetilde{A}_{21} \widetilde{x}_{1}(k)$. As we discussed earlier, here, the matrix $\widetilde{A}_{22}^{\prime}$ is block diagonal, whose blocks are of the size $\left(n_{i}-1\right) \times\left(n_{i}-1\right)$. The inverse of each block can be computed by the corresponding group. The same holds for the third step (22), where the transformation matrices $W_{1}$ and $W_{2}$ are also block diagonal. 
Later in Section 7, through a numerical example, we demonstrate that even with this reduced computation cost, the aggregation-based approach exhibits high performance in convergence rate and accuracy. In particular, two distributed algorithms are compared: One with the original order $n$ and the other with the reduced order $r<n$. The results show that the errors from the true PageRank decrease to comparable levels at similar rates.

\subsection{Aggregated PageRank and its approximation error}

In this subsection, we present two results establishing error bounds for the update scheme in Algorithm 4.2. The results provide useful guidelines on how the aggregation of the web should be done.

The first theorem is based on the sparsity property in the graph $\mathcal{G}$, represented by the node parameter in Assumption 3.1 .

Letting $\epsilon \in(0,1)$ be a parameter that determines the desired level of approximation, we consider the upper bound $\delta$ on node parameters. Aggregate the web so that $\delta$ is sufficiently small that

$$
\delta \leq \frac{m \epsilon}{4(1-m)(1+\epsilon)} .
$$

Theorem 4.5 Under Assumption 3.1 with the parameter $\delta$ satisfying (26), the error between the steady state $x^{\prime}$ in (25) of the update scheme Algorithm 4.2 and the PageRank vector $x^{*}$ is bounded as

$$
\left\|x^{*}-x^{\prime}\right\|_{1} \leq \epsilon
$$

To prove this theorem, it is useful to consider the scheme given by

$$
\left[\begin{array}{c}
\widetilde{x}_{1}(k+1) \\
\widetilde{x}_{2}(k+1)
\end{array}\right]=(1-m) \widetilde{A}^{\prime}\left[\begin{array}{l}
\widetilde{x}_{1}(k) \\
\widetilde{x}_{2}(k)
\end{array}\right]+\frac{m}{n}\left[\begin{array}{l}
u \\
0
\end{array}\right],
$$

where the matrix $\widetilde{A}^{\prime}$ is defined as

$$
\widetilde{A}^{\prime}:=\left[\begin{array}{cc}
\widetilde{A}_{11} & 0 \\
\widetilde{A}_{21} & \widetilde{A}_{22}^{\prime}
\end{array}\right] .
$$

This is a modified version of $\widetilde{A}$ by replacing $\widetilde{A}_{12}$ and $\widetilde{A}_{22}$ with 0 and $\widetilde{A}_{22}^{\prime}$, respectively. Note that the matrix $(1-m) \widetilde{A}^{\prime}$ is stable because by Lemma 4.1, $\widetilde{A}_{11}$ is stochastic and $(1-m) \widetilde{A}_{22}^{\prime}$ is stable. It is straightforward to show that in the scheme (28), the state converges to $\widetilde{x}^{\prime}$ in (24). Then, the vector $x^{\prime}=V^{-1} \widetilde{x}^{\prime}$ given in (25) must be such that

$$
x^{\prime}=(1-m) A^{\prime} x^{\prime}+\frac{m}{n} \mathbf{1}_{n}, \text { where } A^{\prime}:=V^{-1} \widetilde{A}^{\prime} V .
$$

We start with a preliminary result regarding this matrix $A^{\prime}$.

Lemma 4.6 Under Assumption 3.1 , it holds that $\left\|A-A^{\prime}\right\|_{1} \leq 4 \delta$. 
Proof: By the definitions of $\widetilde{A}$ and $\widetilde{A}^{\prime}$, we have $A-A^{\prime}=V^{-1}\left(\widetilde{A}-\widetilde{A^{\prime}}\right) V$. Using the submatrix expressions of (16) and (29) and also (17), we have

$$
A-A^{\prime}=W_{1} \widetilde{A}_{12} V_{2}+W_{2}\left(\widetilde{A}_{22}-\widetilde{A}_{22}^{\prime}\right) V_{2}=\left(W_{1} V_{1}+W_{2} V_{2}\right) A_{\text {ext }} W_{2} V_{2}=A_{\text {ext } 0} W_{2} V_{2},
$$

where the last equality holds by (15) and $W_{1} V_{1}+W_{2} V_{2}=I$. The matrix $W_{1} V_{1}$ is block diagonal in the form of $\operatorname{bdiag}\left(1 / \widetilde{n}_{i} \mathbf{1}_{\widetilde{n}_{i}} \mathbf{1}_{\widetilde{n}_{i}}^{T}\right)$ and is stochastic. Hence, $\left\|W_{1} V_{1}\right\|_{1}=1$. Also, $W_{2} V_{2}=I-W_{1} V_{1}$, which implies $\left\|W_{2} V_{2}\right\|_{1} \leq 2$. Therefore, by Lemma 4.1(ii), the 1-norm of $A-A^{\prime}$ can be bounded as

$$
\left\|A-A^{\prime}\right\|_{1} \leq\left\|A_{\text {exto }}\right\|_{1}\left\|W_{2} V_{2}\right\|_{1} \leq 4 \delta .
$$

Proof of Theorem 4.5: From (21) and (30), it follows that

$$
x^{*}-x^{\prime}=(1-m)\left(A x^{*}-A^{\prime} x^{\prime}\right)=(1-m)\left[\left(A-A^{\prime}\right) x^{*}+A^{\prime}\left(x^{*}-x^{\prime}\right)\right] .
$$

Thus, we have $\left[I-(1-m) A^{\prime}\right]\left(x^{*}-x^{\prime}\right)=(1-m)\left(A-A^{\prime}\right) x^{*}$. Here note that the matrix $I-(1-m) A^{\prime}$ is nonsingular because $(1-m) A^{\prime}$ is stable. Thus, we obtain

$$
x^{*}-x^{\prime}=(1-m)\left[I-(1-m) A^{\prime}\right]^{-1}\left(A-A^{\prime}\right) x^{*} .
$$

By the condition (26) on $\delta$, it also holds that

$$
(1-m)\left\|A^{\prime}\right\|_{1} \leq(1-m)\left[\|A\|_{1}+\left\|A^{\prime}-A\right\|_{1}\right] \leq(1-m)(1+4 \delta)<1,
$$

where the second inequality is due to Lemma 4.6. Hence, from (31), we have

$$
\begin{aligned}
\left\|x^{*}-x^{\prime}\right\|_{1} & \leq(1-m)\left\|\sum_{k=0}^{\infty}(1-m)^{k}\left(A^{\prime}\right)^{k}\left(A-A^{\prime}\right) x^{*}\right\|_{1} \\
& \leq(1-m) \sum_{k=0}^{\infty}\left[(1-m)\left\|A^{\prime}\right\|_{1}\right]^{k}\left\|A-A^{\prime}\right\|_{1}\left\|x^{*}\right\|_{1} \\
& \leq \frac{4 \delta(1-m)}{1-(1-m)(1+4 \delta)} .
\end{aligned}
$$

Finally, by the bound (26) on $\delta$, we obtain the inequality in (27).

The theorem exhibits that aggregation is useful in obtaining a good approximate of the PageRank based on an update scheme of a lower order. For the approximate calculation to be feasible, the critical condition is (i) of Assumption 3.1, setting a limit on the ratio of external links for each group. It is however clear that in the web, many pages have many external links outside its own domain, which will not satisfy this assumption. Such a page should not be grouped with other pages, but instead be treated as a group on its own; these pages will then satisfy (ii) of Assumption 3.1. The proposed aggregation method is closely related to those considered in the context of singular perturbation analyses. The differences will be discussed in detail in Section 5 ,

We proceed to the second result, which also shows how the web aggregation should be carried out from a different perspective. In the proposed scheme, the first step (20) involves only the state 
$\widetilde{x}_{1}$, which consists of the total values of each group. Hence, when the coordinate is transformed back as $x=W \widetilde{x}=W_{1} \widetilde{x}_{1}+W_{2} \widetilde{x}_{2}$ in the third step (22), each entry of its contribution $W_{1} \widetilde{x}_{1}$ represents the average value of the group to which the corresponding page belongs. This means that if the grouping is done so that the values of the members in each group are similar, we can expect that the scheme computes $\widetilde{x}_{1}$ with small error. The following theorem provides a quantitative result on this intuition.

Theorem 4.7 If $\left\|x^{*}-W_{1} \widetilde{x}_{1}^{*}\right\|_{1} \leq \kappa$, then it holds that

$$
\left\|x^{*}-W_{1} \widetilde{x}_{1}^{\prime}\right\|_{1} \leq \frac{\kappa}{m} .
$$

Proof: By (6), we have $x^{*}=W \widetilde{x}^{*}=W_{1} \widetilde{x}_{1}^{*}+W_{2} \widetilde{x}_{2}^{*}$. Thus,

$$
x^{*}-W_{1} \widetilde{x}_{1}^{\prime}=W_{1}\left(\widetilde{x}_{1}^{*}-\widetilde{x}_{1}^{\prime}\right)+W_{2} \widetilde{x}_{2}^{*} .
$$

This means that we shall focus on $\widetilde{x}_{1}^{*}-\widetilde{x}_{1}^{\prime}$. Observe that by definition, $\widetilde{x}_{1}^{*}$ is part of the equilibrium of the recursion in (11) and (12). Also, $\widetilde{x}_{1}^{\prime}$ is the equilibrium of (20). Thus, it follows that

$$
\widetilde{x}_{1}^{*}-\widetilde{x}_{1}^{\prime}=(1-m)\left[\widetilde{A}_{11}\left(\widetilde{x}_{1}^{*}-\widetilde{x}_{1}^{\prime}\right)+\widetilde{A}_{12} \widetilde{x}_{2}^{*}\right] .
$$

Hence, we have $\left[I-(1-m) \widetilde{A}_{11}\right]\left(\widetilde{x}_{1}^{*}-\widetilde{x}_{1}^{\prime}\right)=\widetilde{A}_{12} \widetilde{x}_{2}^{*}$. By Lemma 4.1(i), $\widetilde{A}_{11}$ is a stochastic matrix, and consequently $\rho\left((1-m) \widetilde{A}_{11}\right)=1-m<1$. This implies that $(1-m) \widetilde{A}_{11}$ is a stable matrix, and hence $I-(1-m) \widetilde{A}_{11}$ is nonsingular. As a result, it holds that

$$
\begin{aligned}
\widetilde{x}_{1}^{*}-\widetilde{x}_{1}^{\prime} & =(1-m)\left[I-(1-m) \widetilde{A}_{11}\right]^{-1} \widetilde{A}_{12} \widetilde{x}_{2}^{*}=(1-m) \sum_{k=0}^{\infty}\left[(1-m) \widetilde{A}_{11}\right]^{k} \widetilde{A}_{12} \widetilde{x}_{2}^{*} \\
& =(1-m) \sum_{k=0}^{\infty}\left[(1-m) V_{1} A W_{1}\right]^{k} V_{1} A W_{2} \widetilde{x}_{2}^{*},
\end{aligned}
$$

where in the last equality we used (16). Substitution of (34) into (33) results in

$$
W_{1}\left(\widetilde{x}_{1}^{*}-\widetilde{x}_{1}^{\prime}\right)+W_{2} \widetilde{x}_{2}^{*}=\sum_{k=1}^{\infty}\left\{\left[(1-m)\left(W_{1} V_{1} A\right)\right]^{k}+I\right\} W_{2} \widetilde{x}_{2}^{*} .
$$

Now it follows that

$$
\begin{aligned}
\left\|W_{1}\left(\widetilde{x}_{1}^{*}-\widetilde{x}_{1}^{\prime}\right)+W_{2} \widetilde{x}_{2}^{*}\right\|_{1} & \leq\left\{\left\|\sum_{k=1}^{\infty}\left[(1-m)\left(W_{1} V_{1} A\right)\right]^{k}\right\|_{1}+1\right\}\left\|W_{2} \widetilde{x}_{2}^{*}\right\|_{1} \\
& =\left[\sum_{k=1}^{\infty}\left\|\left[(1-m)\left(W_{1} V_{1} A\right)\right]^{k}\right\|_{1}+1\right]\left\|W_{2} \widetilde{x}_{2}^{*}\right\|_{1} \\
& \leq\left[\sum_{k=1}^{\infty}(1-m)^{k}+1\right] \kappa=\frac{\kappa}{m},
\end{aligned}
$$

where the first equality holds since $W_{1} V_{1} A$ is a stochastic matrix and the second inequality is due to the condition $\left\|W_{2} \widetilde{x}_{2}^{*}\right\|_{1}=\left\|x^{*}-W_{1} \widetilde{x}_{1}^{*}\right\|_{1} \leq \kappa$. Therefore, we arrive at the bound in (32). 


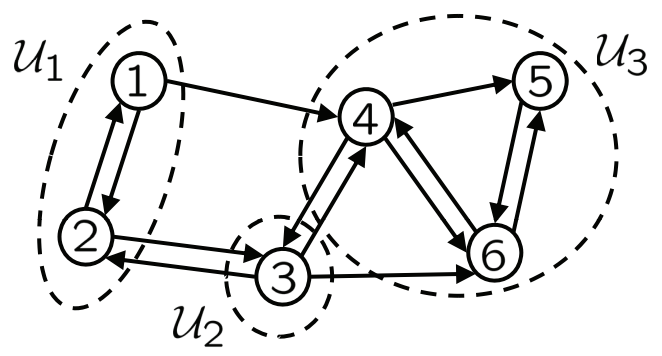

Figure 1: The example web, where the dashed lines indicate the grouping for aggregation

The condition $\left\|x^{*}-W_{1} \widetilde{x}_{1}^{*}\right\|_{1} \leq \kappa$ in the theorem may in general be difficult to check because it requires global information about PageRank. However, it is possible to convert it to a local condition. In fact, a sufficient condition is that, for each page $i \in \mathcal{V}$, the relative error between the value $x_{i}^{*}$ and the average $\left(W_{1} \widetilde{x}_{1}^{*}\right)_{i}$ of its group satisfies $\left|x_{i}^{*}-\left(W_{1} \widetilde{x}_{1}^{*}\right)_{i}\right| \leq \kappa x_{i}^{*}$. Obviously, this relative error is zero for any group with only one member. Thus, for more accurate computations, we may envision to run an algorithm estimating the local value $\left|x_{i}^{*}-\left(W_{1} \widetilde{x}_{1}(k)\right)_{i}\right|$ in real time; if the estimate exceeds a given threshold, then the group should be split into smaller groups, each of which having smaller relative errors. On the other hand, the theorem is stated in terms of the 1-norm of the approximation errors. As we see in the proof, for this particular norm, a fairly tight bound is obtained; the reason is that for column stochastic matrices, the 1-norm is always 1 .

\subsection{Example}

We present a simple example to illustrate the idea of PageRank via web aggregation.

Consider the web consisting of six pages shown in Fig. 1. As a graph, this web is strongly connected. The original link matrix $A$ in (1) is given by

$$
A=\left[\begin{array}{cccccc}
0 & 1 / 2 & 0 & 0 & 0 & 0 \\
1 / 2 & 0 & 1 / 3 & 0 & 0 & 0 \\
0 & 1 / 2 & 0 & 1 / 3 & 0 & 0 \\
1 / 2 & 0 & 1 / 3 & 0 & 0 & 1 / 2 \\
0 & 0 & 0 & 1 / 3 & 0 & 1 / 2 \\
0 & 0 & 1 / 3 & 1 / 3 & 1 & 0
\end{array}\right] .
$$

The PageRank vector $x^{*}$ in (2) can be found as

$$
x^{*}=\left[\begin{array}{llllll}
0.0614 & 0.0857 & 0.122 & 0.214 & 0.214 & 0.302
\end{array}\right]^{T} .
$$

Pages 4 and 6 have the largest number of incoming links, resulting in large PageRank values. Page 6 is more advantageous because the pages contributing to its value via links, i.e., pages 3,4 , and 5 , have larger values than those having links to page 4 . In particular, page 1 has the smallest number of incoming links and obviously the lowest ranking in this web.

We now aggregate the web and partition the nodes into three groups (i.e., $r=3$ ) as $\mathcal{U}_{1}=\{1,2\}$, $\mathcal{U}_{2}=\{3\}$, and $\mathcal{U}_{3}=\{4,5,6\}$. These are indicated by the dashed lines in Fig. 1, and the aggregated 


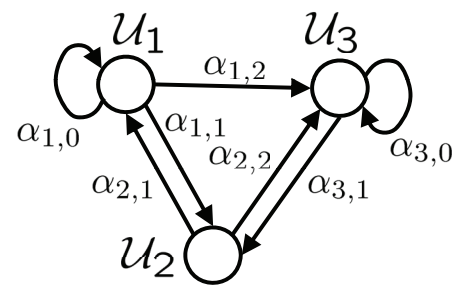

Figure 2: Aggregated graph with the update probabilities $\alpha_{i, \ell}$ for the links (see also Example 6.6)

graph is illustrated in Fig. 2. The nodes $\mathcal{U}_{1}$ and $\mathcal{U}_{3}$ contain self-loops while $\mathcal{U}_{3}$ does not since it is a single group. Here, the node parameters are $\delta_{1}=\delta_{2}=1 / 2, \delta_{3}=1, \delta_{4}=1 / 3$, and $\delta_{5}=\delta_{6}=0$. Thus, all the pages satisfy Assumption 3.1 by taking $\delta=0.5$. This grouping is also reasonable from the viewpoint of Theorem 4.7 because in the true PageRank vector $x^{*}$, the values of the pages in the groups $\mathcal{U}_{1}$ and $\mathcal{U}_{3}$ are relatively close. In this case, the transformation matrix $V$ in (5D) is

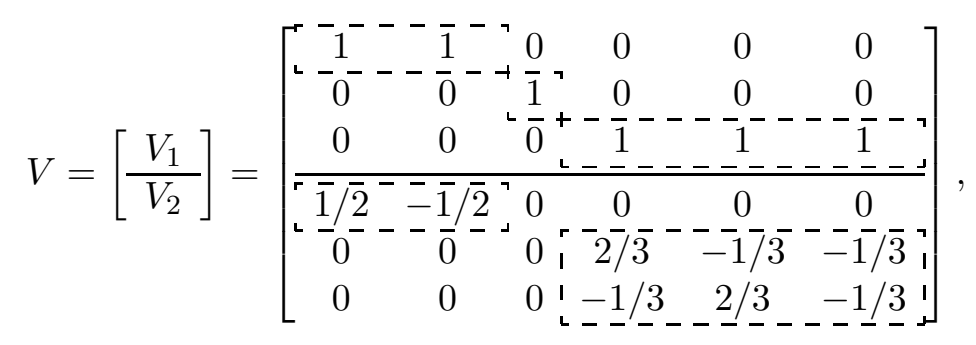

where the diagonal blocks are indicated by dashed-line boxes. In $V_{2}$, the third column corresponding to the single group $\mathcal{U}_{2}$ is zero. Then, the PageRank after the coordinate transformation, $\widetilde{x}^{*}=V x^{*}$, can be found as

$$
\widetilde{x}^{*}=\left[\left(\widetilde{x}_{1}^{*}\right)^{T} \mid\left(\widetilde{x}_{2}^{*}\right)^{T}\right]^{T}=\left[\begin{array}{llllll}
0.147 & 0.122 & 0.731 \mid-0.0121 & -0.0294 & -0.0294
\end{array}\right]^{T} .
$$

Notice that the first state $\widetilde{x}_{1}^{*}$ is a probability vector.

For this grouping, by (13), the matrix $A$ can be decomposed as $A=I+A_{\text {int }}+A_{\text {ext }}$, where the internal matrix $A_{\text {int }}$ and the external matrix $A_{\text {ext }}$ are respectively

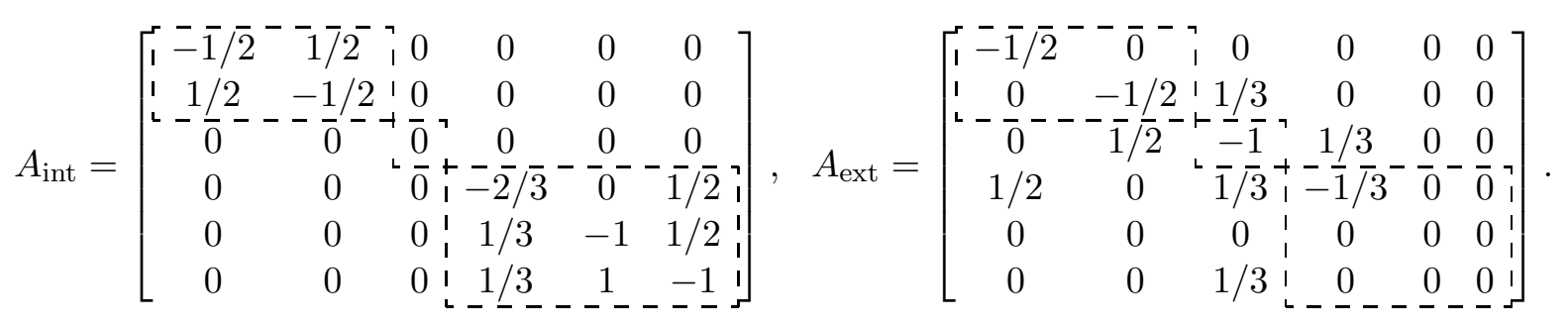

The internal matrix $A_{\text {int }}$ consists of the block-diagonal elements of $A$ while the external matrix $A_{\text {ext }}$ contains the rest. 
The link matrix $\widetilde{A}$ in (10) in the corresponding coordinate becomes

$$
\widetilde{A}=\left[\begin{array}{c|c}
\widetilde{A}_{11} & \widetilde{A}_{12} \\
\hline \widetilde{A}_{21} & \widetilde{A}_{22}
\end{array}\right]=\left[\begin{array}{ccc|ccc}
0.5 & 0.333 & 0 & 0 & 0 & 0 \\
0.25 & 0 & 0.111 & -0.5 & 0.333 & 0 \\
0.25 & 0.667 & 0.889 & 0.5 & -0.333 & 0 \\
\hline 0 & -0.167 & 0 & -0.5 & 0 & 0 \\
0.167 & 0.111 & -0.130 & 0.333 & -0.389 & -0.5 \\
-0.0833 & -0.222 & -0.0185 & -0.167 & -0.0556 & -0.5
\end{array}\right] .
$$

The proposed update scheme in Algorithm 4.2 employs the matrices $\widetilde{A}_{11}$ above and

$$
\left[I-(1-m) \widetilde{A}_{22}^{\prime}\right]^{-1} \widetilde{A}_{21}=\left[\begin{array}{ccc}
0 & -0.167 & 0 \\
0.174 & 0.161 & -0.113 \\
-0.0758 & -0.172 & -0.00177
\end{array}\right],
$$

where $\widetilde{A}_{22}^{\prime}$ is obtained through (17) using $A_{\text {int }}$

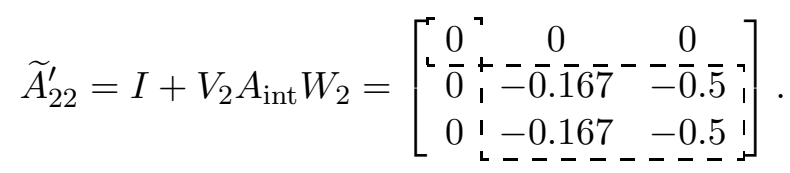

Notice that the matrix $\widetilde{A}_{11}$ is stochastic. Also, $\widetilde{A}_{22}^{\prime}$ has a block-diagonal structure (different from $\left.\widetilde{A}_{22}\right)$ and is a stable matrix. For this scheme, the steady state in the original coordinate is

$$
x^{\prime}=W \widetilde{x}^{\prime}=\left[\begin{array}{llllll}
0.0566 & 0.0920 & 0.125 & 0.212 & 0.213 & 0.302
\end{array}\right]^{T} .
$$

Comparing this with the true value $x^{*}$ in (35), the error is indeed small as $\left\|x^{\prime}-x^{*}\right\|_{1}=0.0188$.

\section{Discussion on aggregation-based methods}

In this section, we provide some discussion on our results from the viewpoint of aggregation. As mentioned in the Introduction, the approach of this paper has been motivated by the singular perturbation results of [2,15] for Markov chains and [7,43] for consensus-like problems with sparse network structures. We have several remarks in relation to these works.

In the Markov chain literature (e.g. [40]), the problem of finding the stationary probability distribution based on aggregation has been long studied; see, for example, [18]. The paper [39] formalizes a general method for finding the exact distribution, and its application to the PageRank computation is discussed in [37]. The approach of [43] can be seen as an interpretation of this method from the viewpoint of singular perturbation.

The papers [2,43] consider the special case when the chain has the so-called nearly completely decomposable structure. In the context of our paper, this means that the external link matrix $A_{\text {ext }}$ in (13) can be bounded as $\left\|A_{\text {ext }}\right\|_{1} \leq \epsilon^{\prime}$ with a small $\epsilon^{\prime}>0$ so that the interaction among different groups is weak. If $\epsilon^{\prime}$ is sufficiently small, the recursion (11) and (12) can be transformed to the singular perturbation form, to which standard results (e.g., [35]) can be applied. Note that in Lemma 4.1 (iii), the bound on the external link matrix is for $A_{\text {ext0 }}$ and not $A_{\text {ext }}$. 
Similarly, the works of [7, 15] deal with problems for multi-agent systems on consensus. The specific setup involves undirected graphs and hence a link matrix which is symmetric and stochastic (i.e., not only column stochastic as in the PageRank problem). In these papers, simple transformation matrices similar to $V$ in (5) have been used. In their results, assumptions are made on the node parameters for all pages and also on the average value of the node parameters for each group; these can be (roughly) stated as $\delta_{i} \leq \delta$ for all $i$ and $\sum_{i \in \mathcal{U}_{j}} \delta_{i} \leq \epsilon^{\prime}$ for all $j$ with $\delta, \epsilon^{\prime}>0$. The consequence is that $\widetilde{A}_{11}$ and $\widetilde{A}_{21}$ can be bounded by constant multiples of $\epsilon^{\prime}$ and hence the problem becomes similar to the Markov chain case mentioned above.

It however is important to note that the web may not have such strong sparsity properties as those assumed in the abovementioned works. For instance, for a page belonging to a small group, one external link can result in a large node parameter. By contrast, the assumption imposed in our approach is the condition $\delta_{i} \leq \delta$ only in the case page $i$ belongs to a group consisting of multiple members; this condition can be checked easily in the grouping procedure outlined in Remark 3.2 . Thus, the results are applicable to a graph with any structure after appropriately grouping the pages. One feature here is the tradeoff between the number $r$ of groups and the node parameter $\delta$ as discussed in Remark 3.2, from Theorem 4.5, we observe that more accurate computation requires a larger number of groups, and thus a smaller $\delta$.

Furthermore, it is emphasized that in the aggregated recursion (20), the link matrix $\widetilde{A}_{11}$ is a stochastic matrix. This is critical in the distributed algorithm in the next section. In contrast, in the singular perturbation form, the corresponding matrix is not necessarily stochastic [2].

\section{Distributed randomized algorithm for aggregated PageRank}

In this section, we construct a distributed randomized scheme for finding the aggregated PageRank in the first step of Algorithm 4.2.

To simplify the notation, we rewrite the aggregated PageRank in (24) as $\xi^{\prime}:=\widetilde{x}_{1}^{\prime}$ and moreover the recursion in the first step (20) as

$$
\xi(k+1)=(1-m) \Phi \xi(k)+\frac{m}{n} u,
$$

where the link matrix is denoted by $\Phi=\left(\phi_{i j}\right):=\widetilde{A}_{11}$ and the state by $\xi(k):=\widetilde{x}_{1}(k)$.

The objective is to compute the aggregated PageRank $\xi^{\prime}$ via the distributed update scheme of (36) in the form given by

$$
\xi(k+1)=(1-\hat{m}) \Phi_{\eta(k)} \xi(k)+\frac{\hat{m}}{n} u,
$$

where $\xi(k) \in \mathbb{R}^{r}$ is the state whose initial condition $\xi(0)$ is a probability vector, and $\hat{m} \in(0,1)$; the process $\eta(k):=\left[\eta_{1}(k) \cdots \eta_{r}(k)\right]$ defined in (7) determines the communication pattern at time $k$. In this scheme, each group $i$ also computes the time average of its own state $\xi_{i}$. Let $\psi(k)$ be the average of $\xi(0), \ldots, \xi(k)$ as

$$
\psi(k)=\frac{1}{k+1} \sum_{\ell=0}^{k} \xi(\ell)=\frac{1}{k+1}(k \psi(k-1)+\xi(k)) .
$$


Let $\alpha \in(0,1]$, which is called the base probability. Recall that the update probability $\alpha_{i, \ell}$ in (8) and (9) determines the probability that group $i$ transmits to its neighbors belonging to $\widetilde{\mathcal{V}}_{i, \ell}$ for $\ell \neq 0$. Assume that they are chosen so that

$$
\alpha_{i, \ell} \in\left[\alpha \sum_{j \in \widetilde{\mathcal{V}}_{i, \ell}} \phi_{j i}, 1\right], \quad \ell=1,2, \ldots, g_{i}, \quad \sum_{\ell=0}^{g_{i}} \alpha_{i, \ell}=1 .
$$

It is noted that by Lemma 4.1 (i), the link matrix $\Phi=\widetilde{A}_{11}$ is stochastic, and thus $\sum_{j=1}^{r} \phi_{j i}=1$. Hence, in this condition, the lower bound $\alpha \sum_{j \in \widetilde{\mathcal{V}}_{i, \ell}} \phi_{j i}$ on $\alpha_{i, \ell}$ is at most $\alpha$.

In (37), the distributed link matrices $\Phi_{q_{1}, \ldots, q_{r}}$ for $q_{i} \in\left\{0,1, \ldots, g_{i}\right\}, i \in \widetilde{\mathcal{V}}$, are given by

$$
\left(\Phi_{q_{1}, \ldots, q_{r}}\right)_{p i}:= \begin{cases}\frac{\alpha}{\alpha_{i, \ell}} \phi_{p i} & \text { if } q_{i}=\ell \neq 0, p \in \widetilde{\mathcal{V}}_{i, \ell}, \\ 1-\frac{\alpha}{\alpha_{i, \ell}} \sum_{j \in \widetilde{\mathcal{V}}_{i, \ell}} \phi_{j i} & \text { if } q_{i}=\ell \neq 0, p=i, \\ 1 & \text { if } q_{i}=0, p=i, \\ 0 & \text { otherwise }\end{cases}
$$

for $p, i \in \widetilde{\mathcal{V}}$. Notice that these link matrices are in accordance with the communication pattern specified by $\eta(k)$, i.e., $\left(\Phi_{\eta(k)}\right)_{p i}>0$ if and only if group $i$ sends its value to group $p$ at time $k$.

Then, we can establish some desired properties of the link matrices for the update scheme (37) to converge. These facts are stated in the proposition below.

Proposition 6.1 For the distributed link matrices $\Phi_{q}$ in (40), the following two properties are satisfied:

(i) For each $q$, the matrix $\Phi_{q}$ is stochastic.

(ii) The average matrix $\bar{\Phi}:=E\left[\Phi_{\eta(k)}\right]$ can be written as $\bar{\Phi}=\alpha \Phi+(1-\alpha) I$.

Proof: (i) Let $\phi_{i} \in \mathbb{R}^{r}$ be the $i$ th column of $\Phi$ and further let $\phi_{i}^{(\ell)} \in \mathbb{R}^{r}$ be a vector containing only those elements corresponding to the nodes in $\widetilde{\mathcal{V}}_{i, \ell}$, i.e., $\left(\phi_{i}^{(\ell)}\right)_{p}:=\phi_{p i}$ if $p \in \widetilde{\mathcal{V}}_{i, \ell}$ and 0 otherwise for $\ell=1,2, \ldots, g_{j}$ and $p, i \in \widetilde{\mathcal{V}}$. Notice that

$$
\phi_{i}=\sum_{\ell=1}^{g_{i}} \phi_{i}^{(\ell)} .
$$

Now, the $i$ th column of $\Phi_{q}$ depends only on $q_{i}$; so denoting this column by $\widetilde{\phi}_{i}^{(\ell)}$ with $\ell=q_{i}$, we have by (40)

$$
\widetilde{\phi}_{i}^{(\ell)}= \begin{cases}\frac{\alpha}{\alpha_{i, \ell}} \phi_{i}^{(\ell)}+\left(1-\frac{\alpha}{\alpha_{i, \ell}} \sum_{j \in \widetilde{\mathcal{V}}_{i, \ell}} \phi_{j i}\right) e_{i} & \text { if } \ell \in\left\{1, \ldots, g_{i}\right\}, \\ e_{i} & \text { if } \ell=0\end{cases}
$$

where $e_{j} \in \mathbb{R}^{r}$ is the unit vector whose $j$ th element is 1 and the rest are 0 . It is now clear that $\widetilde{\phi}_{i}^{(\ell)} \geq 0$ because by definition $\phi_{i}^{(\ell)} \geq 0$ and $1-\alpha / \alpha_{i, \ell} \sum_{j \in \widetilde{\mathcal{V}}_{i, \ell}} \phi_{j i} \geq 0$ by the choice of $\alpha_{i, \ell}$ in (39). Moreover, if $\ell \neq 0$, it follows that

$$
\left\|\widetilde{\phi}_{i}^{(\ell)}\right\|_{1}=\frac{\alpha}{\alpha_{i, \ell}}\left\|\phi_{i}^{(\ell)}\right\|_{1}+\left(1-\frac{\alpha}{\alpha_{i, \ell}} \sum_{j \in \widetilde{\mathcal{V}}_{i, \ell}} \phi_{j i}\right)=1,
$$


where we used the fact $\left\|\phi_{i}^{(\ell)}\right\|_{1}=\sum_{j \in \widetilde{\mathcal{V}}_{i, \ell}} \phi_{j i}$. It thus follows that each column of $\Phi_{q}$ is nonnegative and the sum of the elements equals one; this implies that this matrix $\Phi_{q}$ is stochastic.

(ii) Let $\bar{\phi}_{i}$ be the $i$ th column of the average matrix $\bar{\Phi}$. By the distribution of $\eta(k)$, it holds that

$$
\bar{\phi}_{i}=E\left[\widetilde{\phi}_{i}^{\left(\eta_{i}(k)\right)}\right]=\sum_{\ell=0}^{g_{i}} \alpha_{i, \ell} \widetilde{\phi}_{i}^{(\ell)}=\alpha \sum_{\ell=1}^{g_{i}}\left(\phi_{i}^{(\ell)}-\sum_{j \in \widetilde{\mathcal{V}}_{i, \ell}} \phi_{j i} e_{i}\right)+e_{i} .
$$

By (41) and stochasticity of $\Phi$, we have $\bar{\phi}_{i}=\alpha \phi_{i}+(1-\alpha) e_{i}$. This holds for $i=1, \ldots, r$, and consequently we obtain $\bar{\Phi}=\alpha \Phi+(1-\alpha) I$.

We next show that the aggregated PageRank vector $\xi^{\prime}$ can be expressed in terms of the distributed link matrices. Recall that by definition, $\xi^{\prime}=\widetilde{x}_{1}^{\prime}$. From (23), we have that $\xi^{\prime}$ is the unique eigenvector of the the link matrix given by $\Gamma:=(1-m) \Phi+(m / n) u \mathbf{1}_{r}$ with the property $\mathbf{1}_{r}^{T} \xi^{\prime}=1$. Due to the proposition above, this characterization can be extended using the distributed link matrices $\Phi_{\eta(k)}$. Define the modified link matrices by $\Gamma_{\eta(k)}:=(1-\hat{m}) \Phi_{\eta(k)}+(\hat{m} / n) u \mathbf{1}_{r}^{T}$, and their average by

$$
\bar{\Gamma}:=E\left[\Gamma_{\eta(k)}\right]=(1-\hat{m}) \bar{\Phi}+\frac{\hat{m}}{n} u \mathbf{1}_{r}^{T}
$$

Take the parameter $\hat{m}$ as

$$
\hat{m}:=\frac{m \alpha}{1-(1-\alpha) m} .
$$

The following lemma is the aggregated version of Lemma 3.3 in [30].

Lemma 6.2 For the parameter $\hat{m}$ given in (42), we have the following:

(i) $\hat{m} \in(0,1)$ and $\hat{m}<m$.

(ii) $\bar{\Gamma}=\frac{\hat{m}}{m} \Gamma+\left(1-\frac{\hat{m}}{m}\right) I$.

(iii) The aggregated PageRank vector $\xi^{\prime}=\widetilde{x}_{1}^{\prime}$ in (23) is the unique eigenvector of the average matrix $\bar{\Gamma}$ corresponding to the eigenvalue 1 .

Remark 6.3 It is interesting that the choice of $\hat{m}$ in (42) is different from $m$, but is critical in establishing the lemma. In particular, noting that the average matrix $\bar{\Gamma}$ is stochastic, we can guarantee that the average state $\bar{\xi}(k):=E[\xi(k)]$ of the update scheme in (37) converges to the desired vector $\xi^{\prime}$. This is because it follows the recursion

$$
\bar{\xi}(k+1)=\bar{\Gamma} \bar{\xi}(k),
$$

where $\bar{\xi}(0)$ is a probability vector. We however emphasize that the state $\xi(k)$ itself does not converge to the aggregated PageRank $\xi^{\prime}$. To resolve this issue, it turns out to be essential to introduce the time average $\psi(k)$ as we see next.

We are in the position to derive a convergence result for the distributed scheme (37) under the probability allocation in (8) for the linked nodes. 
Theorem 6.4 Consider the distributed update scheme in (37) and (38). For any update probabilities $\alpha_{i, \ell} \in(0,1], i \in \widetilde{\mathcal{V}}, \ell \in\left\{0,1, \ldots, g_{i}\right\}$, satisfying the conditions in (39), the aggregated PageRank $\xi^{\prime}$ can be obtained from the time average $\psi(k)$ of the states $\xi(k)$ in the mean-square sense as $E\left[\left\|\psi(k)-\xi^{\prime}\right\|^{2}\right] \rightarrow 0, k \rightarrow \infty$.

The proof of this theorem follows from noticing that, in Theorem 3.4 of [30], to establish the mean-square convergence, the properties in Proposition 6.1 are sufficient. In other words, for convergence, only stochasticity of the link matrices and the average behavior of the update scheme are relevant. The type of convergence guaranteed by the theorem is known as ergodicity for stochastic processes [42]. While the general results of [17] can be applied for the proof, we have developed in [30] a more specific one, which have been useful in extending the algorithm to incorporate a stopping criterion there. It was also shown there that the convergence rate is of order $1 / k$ due to the time averaging.

The distributed update scheme presented above has the following features: (i) The computation performed at each group $i$ includes the updates in the state $\xi_{i}$ in (37) and the time average $\psi_{i}$ in (38). (ii) The communication among the groups is local in the sense that each group communicates only over direct outgoing links, as seen from the link matrices in (40). (iii) The amount of communication is determined by the process $\eta$, which specifies the pattern in the interaction between the pages. (iv) At any group, the update probabilities $\alpha_{i, \ell}$ can be allocated to linked groups locally without information exchange among groups; one global parameter is $\alpha$, which is critical for the convergence of the proposed algorithm 5 .

Remark 6.5 Compared to the original scheme in [30], a significant advantage of the one above is that the nodes need to communicate with neighbors only over outgoing links. The identity of such links is necessarily contained in their local data. This is observed in the link matrices $\Phi_{q}$ in (40) where only the columns of $\Phi$ (and not the rows) corresponding to the indices $q_{i}$ taking values 1 are used. In contrast, in [30], the protocol is that the nodes send data over incoming links as well. Consequently, we also stress that the implementation of this algorithm is simple. A closer look at the matrices $\Phi_{q}$ suggests us that group $j$ should just send its current value with some weight to the linked groups, where their values are updated by simply adding up the values received at the time. Hence, no memory is needed for storing values of other groups. This characteristic is realized by the assumption on stochasticity of the distributed link matrices. In contrast, such memory is necessary in algorithms using asynchronous iteration [6, 20, 36]. In fact, the most recently received values of all groups having links to the group need to be stored. Hence, the memory size is determined by the number of incoming links and may be large for popular groups.

The convergence rate of this scheme can be discussed from the viewpoint of its average dynamics. As mentioned in Remark 6.3, the average state $\bar{\xi}(k)$ converges to the aggregated PageRank $\xi^{\prime}$.

\footnotetext{
${ }^{5}$ Practical issues related to implementation of the scheme are outside the scope of this paper. Clearly, for the PageRank values reported by page owners to be trusted, some regulations must be enforced. Also, reliability of the rankings can be affected by links purposefully added to increase PageRank of certain pages; some works have reported methods to detect such web spamming (e.g., 3, 37). Further discussions on this point are given in the footnote of the Introduction.
} 
Because of the recursion (43), the asymptotic rate of convergence is exponential and is dominated by the the second largest eigenvalue $\lambda_{2}(\bar{\Gamma})$ of $\bar{\Gamma}$ in magnitude. By Lemma 6.2 (ii), this eigenvalue can be bounded as

$$
\left|\lambda_{2}(\bar{\Gamma})\right|=\frac{\hat{m}}{m}\left|\lambda_{2}(\Gamma)\right|+1-\frac{\hat{m}}{m} \leq \frac{1-m}{1-(1-\alpha) m},
$$

where the inequality holds because $\left|\lambda_{2}(\Gamma)\right| \leq 1-m$; see [37]. Therefore, the convergence rate depends on the base probability $\alpha$ in communication, i.e., more communication implies faster convergence. On the other hand, it is interesting to observe that the bound (44) above is independent of the choices of the individual update probabilities $\alpha_{i, \ell}$ as well as the number $r$ of groups.

For the update probabilities $\alpha_{i, \ell}$ to satisfy the conditions (39), one possible choice is the following:

$$
\alpha_{i, \ell}=\left\{\begin{array}{ll}
1-\alpha & \text { if } \ell=0, \\
\alpha \frac{\sum_{j \in \tilde{\mathcal{V}}_{i, \ell} \phi_{j i}}}{\sum_{j \in \tilde{\mathcal{V}}_{i} \phi_{j i}}} & \text { if } \ell=1, \ldots, g_{i},
\end{array} \quad \text { for } i \in \widetilde{\mathcal{V}}_{i} .\right.
$$

In this case, the probability for group $i$ to transmit information to some neighbor is in total equal to the base probability: $\sum_{\ell=1}^{g_{i}} \alpha_{i, \ell}=\alpha$. Further, the probability for communicating with group in $\tilde{\mathcal{V}}_{i, \ell}$ is proportional to the weights $\sum_{j \in \widetilde{\mathcal{V}}_{i, \ell}} \phi_{j i}$ of the corresponding entries in the $i$ th column of the link matrix $\Phi$. Hence, the frequency of communication among groups with more links is higher.

Example 6.6 We study the distributed algorithm based on the aggregated web in Fig. 2 from Section 4.3. Let the communication be such that each group communicates with the neighbors separately and the update probabilities $\alpha_{i, \ell}$ are chosen as in (45) above. Thus, for example, group 1 has two neighbor sets given by $\widetilde{\mathcal{V}}_{1,1}=\{2\}$ and $\widetilde{\mathcal{V}}_{1,2}=\{3\}$. Their update probabilities will become $\alpha_{1,1}=\alpha_{1,2}=\alpha / 2$, resulting in the probability of no communication to be $\alpha_{1,0}=1-\alpha$; these probabilities are indicated in Fig. 2. Group 2 also has two links, so let $\widetilde{\mathcal{V}}_{2,1}=\{1\}$ and $\widetilde{\mathcal{V}}_{2,2}=\{3\}$. Similarly to the case above, let the update probabilities be $\alpha_{2,0}=1-\alpha, \alpha_{2,1}=\alpha / 3$, and $\alpha_{2,2}=2 \alpha / 3$. Finally, the only link of group 3 is to group 2 , which forms the group $\widetilde{\mathcal{V}}_{3,1}=\{2\}$. We can take $\alpha_{3,0}=1-\alpha$ and $\alpha_{3,1}=\alpha$.

The distributed link matrices $\Phi_{q_{1}, q_{2}, q_{3}}$ in (40) can be expressed as

$$
\Phi_{q_{1}, q_{2}, q_{3}}=\left[\begin{array}{lll}
\widetilde{\phi}_{1}^{\left(q_{1}\right)} & \widetilde{\phi}_{2}^{\left(q_{2}\right)} & \widetilde{\phi}_{3}^{\left(q_{3}\right)}
\end{array}\right], \quad q_{1}, q_{2} \in\{0,1,2\}, q_{3} \in\{0,1\},
$$

where each column is given as

$$
\begin{aligned}
& \widetilde{\phi}_{1}^{(0)}=\left[\begin{array}{l}
1 \\
0 \\
0
\end{array}\right], \widetilde{\phi}_{1}^{(1)}=\left[\begin{array}{c}
1 / 2 \\
1 / 2 \\
0
\end{array}\right], \widetilde{\phi}_{1}^{(2)}=\left[\begin{array}{c}
1 / 2 \\
0 \\
1 / 2
\end{array}\right], \\
& \widetilde{\phi}_{2}^{(0)}=\left[\begin{array}{l}
0 \\
1 \\
0
\end{array}\right], \widetilde{\phi}_{2}^{(1)}=\left[\begin{array}{l}
1 \\
0 \\
0
\end{array}\right], \widetilde{\phi}_{2}^{(2)}=\left[\begin{array}{l}
0 \\
0 \\
1
\end{array}\right], \\
& \widetilde{\phi}_{3}^{(0)}=\left[\begin{array}{l}
0 \\
0 \\
1
\end{array}\right], \widetilde{\phi}_{3}^{(1)}=\left[\begin{array}{c}
0 \\
1 / 9 \\
8 / 9
\end{array}\right] .
\end{aligned}
$$




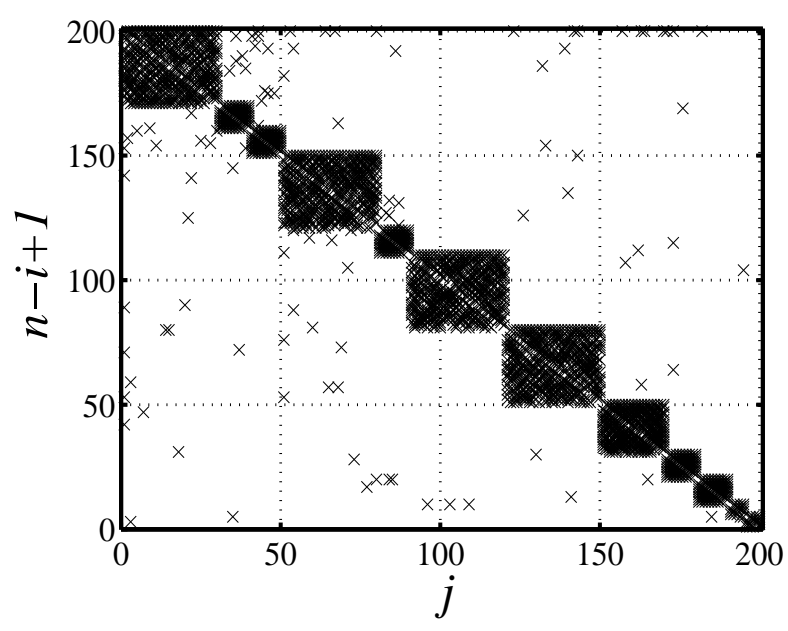

Figure 3: Connectivity matrix with a block diagonal structure

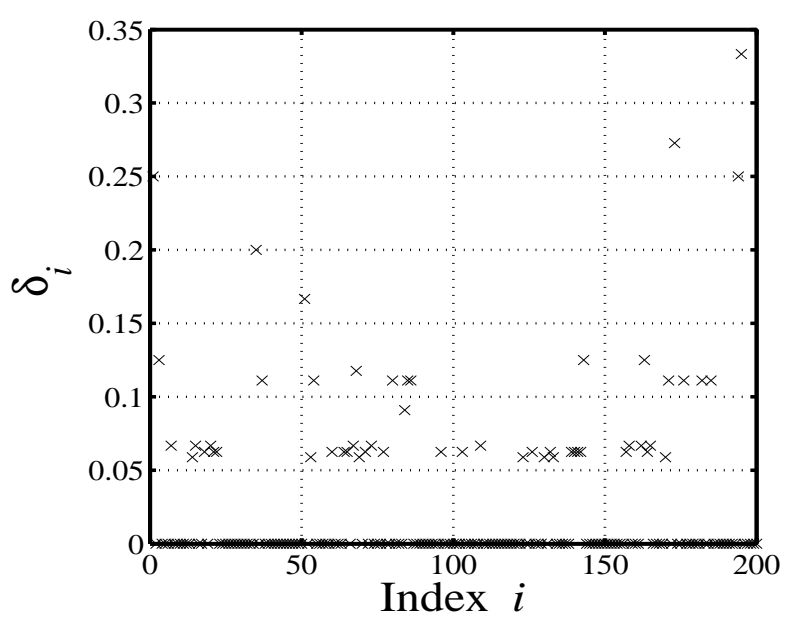

Figure 4: The original node parameters $\delta_{i}$ of the pages

It is straightforward to check that the properties shown in Proposition 6.1 hold: Each $\Phi_{q_{1}, q_{2}, q_{3}}$ is a stochastic matrix and the average matrix $\bar{\Phi}$ satisfies the relation $\bar{\Phi}=\alpha \Phi+(1-\alpha) I$.

\section{$7 \quad$ Numerical example}

In this section, we provide a numerical example to illustrate the aggregation-based approach.

We consider a web with 200 pages $(n=200)$ whose link structure was randomly generated with some level of sparsity. The connectivity matrix representing the links is shown in Fig. 3, where the mark $\times$ indicates the presence of a link from page $j$ to $i$. Here, we made 12 dense diagonal blocks, each of which consisting of 5 to 30 pages. Further, there are two less dense blocks of pages 1 to 50 and pages 51 to 90 . These blocks are initially considered as groups. The first page in each dense block has links from all other pages in the same block, and in particular, pages 1 and 51 are given more incoming and/or outgoing links; these pages will have high PageRank values as we will see.

Following the grouping procedure outlined in Remark 3.2, we first computed the node parameters $\delta_{i}$ for each page $i$ with respect to the groups described above; these are plotted in Fig. 4. This parameter can be large when the corresponding page has many outgoing links as pages 1 and 51 . It can also be large when a page is in a small group, but has external links; this is the case with pages 173, 194, and 195. However, many pages have no external links, and hence the average value of $\delta_{i}$ is relatively small at 0.0260 . We remark that this average is similar to the value reported in [10], which is found from real web data when pages are grouped according to the hosts.

The grouping procedure is determined by the bound $\delta$ on node parameters. The relation between $\delta$ and the number $r$ of groups is shown in Fig. 5. The line in the figure is not necessarily nonincreasing and is in fact piecewise constant. On the other hand, Fig. 6 exhibits the relation between $\delta$ and the error in the approximated PageRank measured by $\left\|x^{\prime}-x^{*}\right\|_{1}$. These two figures 


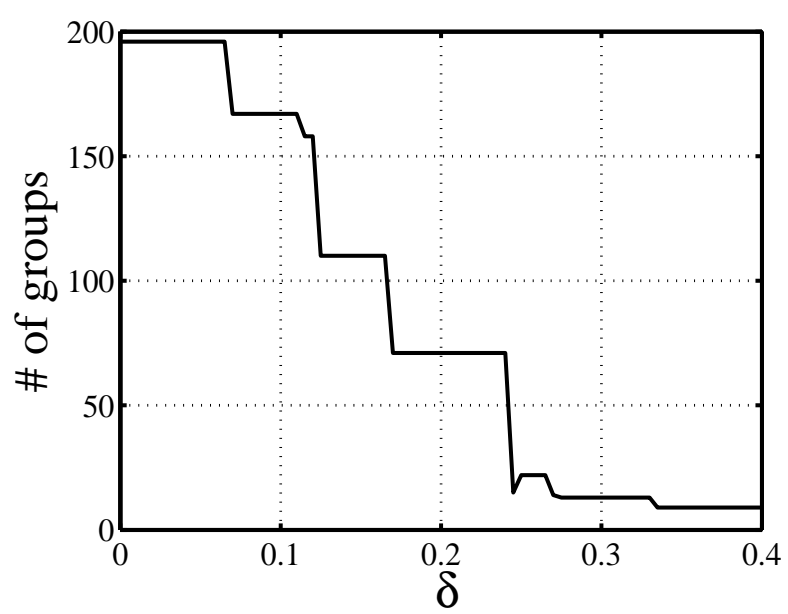

Figure 5: The number $r$ of groups versus $\delta$

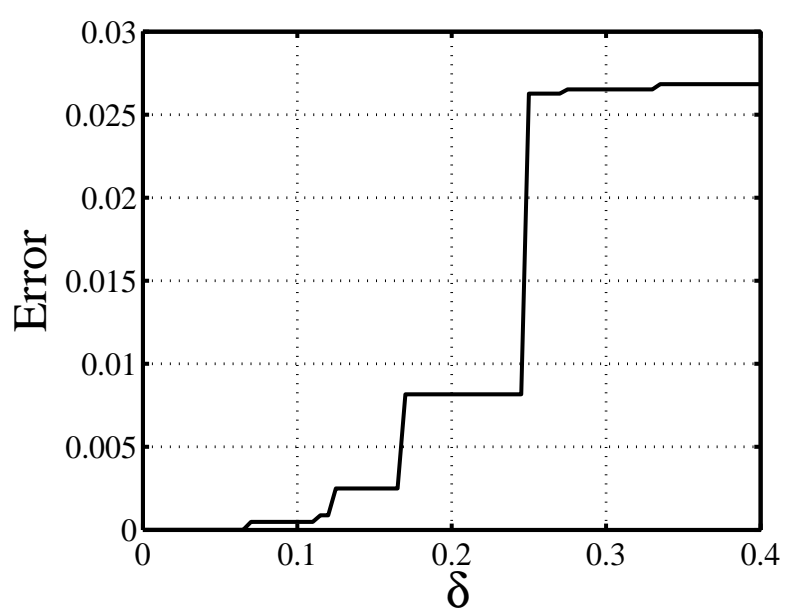

Figure 6: Error $\left\|x^{\prime}-x^{*}\right\|_{1}$ in PageRank versus $\delta$

clearly show the tradeoff between accuracy in computation and the size of the problem: Smaller $\delta$ implies smaller error but larger number $r$ of groups.

To see the errors in the PageRank of the individual pages, we plotted in Fig. 7 the true values $x_{i}^{*}$ and the approximated values $x_{i}^{\prime}$ for $i \in \mathcal{V}$ when $\delta=0.2$ is used by $\times$ and $\bigcirc$, respectively. This computation was done with 71 groups $(r=71)$. We observe that the error is small in general.

Finally, we applied the distributed randomized algorithm (37) and (38) for computing the aggregated PageRank $\xi^{\prime}$ and then the entire PageRank vector $x$. Here, for the gossip communication protocol, the grouping $\widetilde{\mathcal{V}}_{i, \ell}, i=1, \ldots, r, \ell=0,1, \ldots, g_{i}$, of the neighbors is based on the original groups in the block structure of the connectivity matrices. Furthermore, we set the update probabilities $\alpha_{i, \ell}$ using the formula in (45) with the base probability $\alpha=0.5$. In Fig. 8 , sample paths of the time average $\psi_{i}(k)$ for groups 50 to 59 are shown in solid lines and the true values $\xi_{i}^{\prime}$ of the aggregated PageRank in dashed lines. It is clear that the time average converges to the true value.

We would like to emphasize that the convergence performance of this reduced-order scheme is similar to the case without aggregation. To make a fair comparison, we computed the state $x(k)$ obtained from the time average $\psi_{i}(k)$ and then its overall error from the true PageRank $x^{*}$. We plotted in Fig. 9 the 1-norm of $x(k)-x^{*}$ by the solid line. Then, the same error was computed from the time average of the full-order scheme, which can be obtained by setting the node parameter very small as $\delta=0.01$; the result is shown by the dashed line in the same plot. The convergence rates as well as the achieved error levels at the final time are comparable for the two cases. The reduced-order case is clearly advantageous since it requires less operation as we have discussed in Remark 4.4. More concretely, in the example considered here, the numbers of nonzero entries for the link matrices $A \in \mathbb{R}^{200 \times 200}$ and $\widetilde{A}_{11}=\Phi \in \mathbb{R}^{71 \times 71}$ are, respectively, $f_{0}(A)=2623$ and $f_{0}\left(\widetilde{A}_{11}\right)=780$. In view of Table 1, the difference in computation costs is evident. 


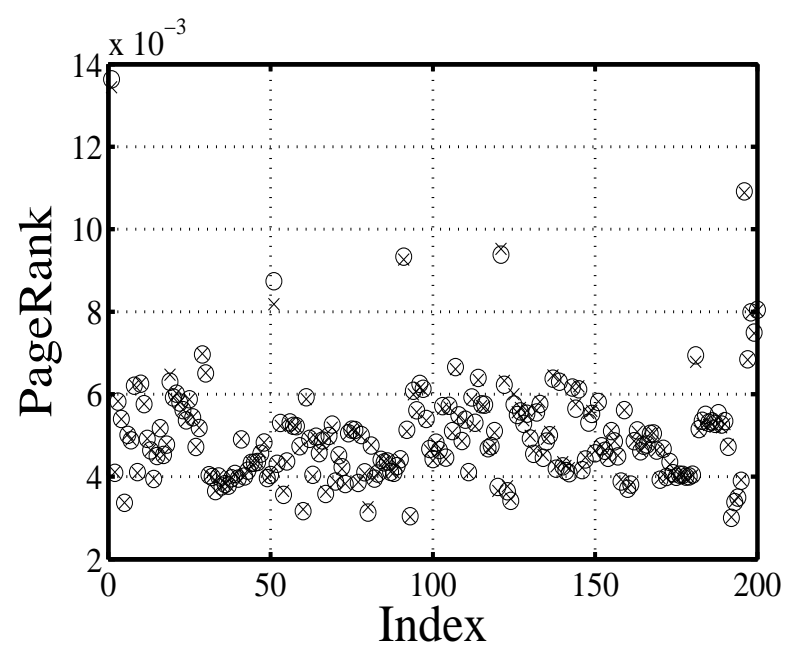

Figure 7: PageRank: True values $x_{i}^{*}($ in $\times)$ and approximation $x_{i}^{\prime}$ with $\delta=0.2$ (in $\bigcirc$ ) for $i=$ $1, \ldots, n$

\section{Conclusion}

In this paper, we have developed a distributed randomized algorithm for obtaining the PageRank values which performs well with reduced computation and communication loads. The approach is based on a novel aggregation technique of the web. First, we have proposed a simple procedure for grouping pages under the criterion of node parameters. Then, the notion of aggregated PageRank has been introduced, based on which approximates of the true values for individual pages can be computed. Error bounds on the approximation level have been derived. Moreover, we have developed the distributed randomized algorithm of lower order for the computation of aggregated PageRank. The advantage of the approach in terms of computation cost as well as convergence properties have been discussed in detail and also demonstrated by a numerical example.

In the future, we will further study aggregation-based methods for PageRank to improve the convergence rate of the update scheme and the effects of incorporating multi-level groupings. More generally, for large-scale systems, the concept of aggregation is of great importance and may provide useful tools for other interesting problems such as smart grid for power distribution.

Acknowledgement: The authors would like to thank Athanasios C. Antoulas, Fabrizio Dabbene, Soura Dasgupta, Shinji Hara, and Jun-ichi Imura for helpful discussions.

\section{References}

[1] Special section on complex networked control systems. IEEE Control Systems Magazine, 27(4), 2007.

[2] R. W. Aldhaheri and H. K. Khalil. Aggregation of the policy iteration method for nearly completely decomposable Markov chains. IEEE Trans. Autom. Control, 36:178-187, 1991.

[3] R. Andersen, C. Borgs, J. Chayes, J. Hopcroft, V. S. Mirrokni, and S.-H. Teng. Local computation of PageRank contributions. In Algorithms and Models for the Web-Graph, volume 4863 of Lect. Notes Comp. Sci., pages 150-165. Springer, Berlin, 2007. 


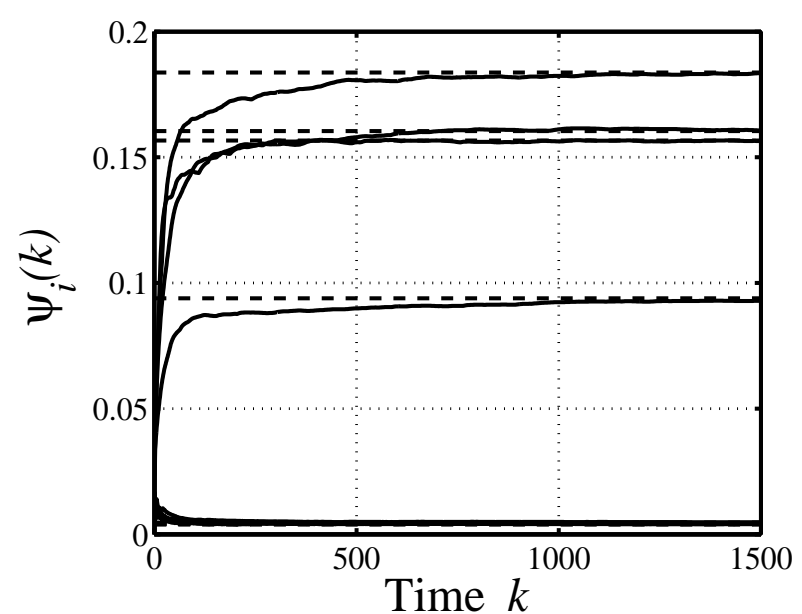

Figure 8: Distributed randomized algorithm for aggregated PageRank: Sample paths of $\psi_{i}(k)$ with $\delta=0.2$ (solid lines) and the true values $\xi_{i}^{\prime}$ (dashed lines) for $i=50, \ldots, 59$

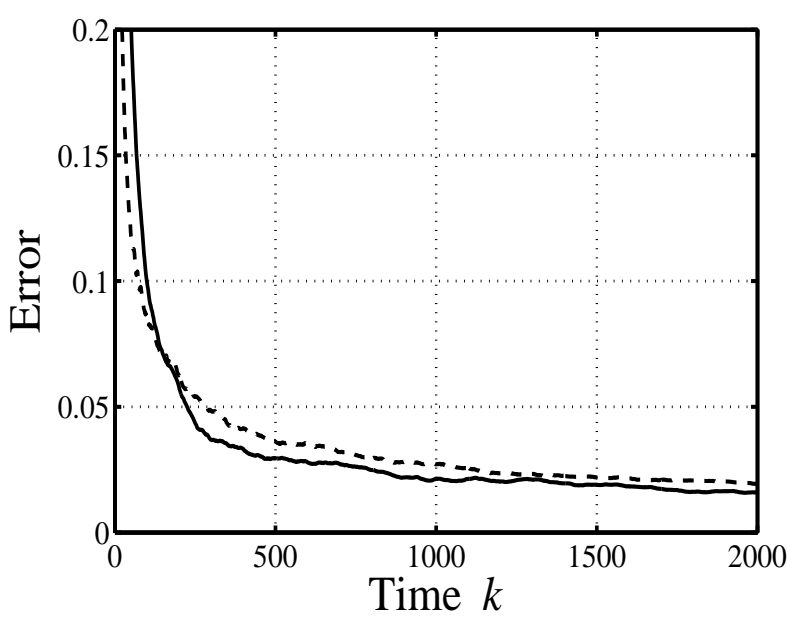

Figure 9: Comparison of distributed randomized algorithms: Sample paths of the error $\left\|x(k)-x^{*}\right\|_{1}$ of the aggregated scheme with $\delta=0.2$ (solid line) and the full-order scheme with $n=200$ (dashed line)

[4] P. J. Antsaklis and J. Baillieul, Guest Editors. Special Issue on the Technology of Networked Control Systems. Proc. IEEE, 95(1), 2007.

[5] K. Avrachenkov, N. Litvak, D. Nemirovsky, and N. Osipova. Monte Carlo methods in PageRank computation: When one iteration is sufficient. SIAM J. Numer. Anal., 45:890-904, 2007.

[6] D. P. Bertsekas and J. N. Tsitsiklis. Parallel and Distributed Computation: Numerical Methods. PrenticeHall, Englewood Cliffs, NJ, 1989.

[7] E. Biylk and M. Arcak. Area aggregation and time-scale modeling for sparse nonlinear networks. Systems 83 Control Letters, 57:142-149, 2008.

[8] S. Boyd, A. Ghosh, B. Prabhakar, and D. Shah. Randomized gossip algorithms. IEEE Trans. Inform. Theory, 52:2508-2530, 2006.

[9] S. Brin and L. Page. The anatomy of a large-scale hypertextual Web search engine. Computer Networks \& ISDN Systems, 30:107-117, 1998.

[10] A. Z. Broder, R. Lempel, F. Maghoul, and J. Pedersen. Efficient PageRank approximation via graph aggregation. Inform. Retrieval, 9:123-138, 2006.

[11] K. Bryan and T. Leise. The $\$ 25,000,000,000$ eigenvector: The linear algebra behind Google. SIAM Rev., 48:569-581, 2006.

[12] K. Cai and H. Ishii. Quantized consensus and averaging on gossip digraphs. IEEE Trans. Autom. Control, 56:2087-2100, 2011.

[13] R. Carli, F. Fagnani, A. Speranzon, and S. Zampieri. Communication constraints in the average consensus problem. Automatica, 44:671-684, 2008.

[14] D. Chaffey, C. Lake, and A. Friedlein. Search Engine Optimization: Best Practice Guide. E-consultancy, 2009.

[15] J. H. Chow and P. V. Kokotovic. Time scale modeling of sparse dynamic networks. IEEE Trans. Autom. Control, 30:714-722, 1985. 
[16] M. Coderch, A. S. Willsky, S. S. Sastry, and D. A. Castanon. Hierarchical aggregation of singularly perturbed finite state Markov chain processes. Stochastics, 8:259-289, 1983.

[17] R. Cogburn. On products of random stochastic matrices. Contemporary Mathematics, 50:199-213, 1986.

[18] P. J. Coutois. Error analysis in nearly-completely decomposable stochastic systems. Econometrica, 43:691-709, 1975.

[19] B. C. Csáji, R. M. Jungers, and V. D. Blondel. PageRank optimization by edge selection. Discrete Applied Mathematics, to appear, 2011.

[20] D. V. de Jager and J. T. Bradley. Asynchronous iterative solution for state-based performance metrics. In Proc. ACM SIGMETRICS, pages 373-374, 2007.

[21] C. de Kerchove, L. Ninove, and P. Van Dooren. Influence of the outlinks of a page on its PageRank. Linear Algebra and its Applications, 429:1254-1276, 2008.

[22] J.-C. Delvenne, S. Yaliraki, and M. Barahona. Stability of graph communities across time scales. Proc. Natl. Acad. Sci. USA, 107:12755-12760, 2010.

[23] K. Deng, P. G. Mehta, and S. P. Meyn. A simulation-based method for aggregating Markov chains. In Proc. 48th IEEE Conf. on Decision and Control and 28th Chinese Control Conf., pages 4710-4716, 2009.

[24] F. Fagnani and S. Zampieri. Randomized consensus algorithms over large scale networks. IEEE J. on Selected Areas of Communications, 26:634-649, 2008.

[25] G. H. Golub and C. F. Van Loan. Matrix Computations, 3rd edition. Johns Hopkins University Press, 1996.

[26] Y. Hatano and M. Mesbahi. Agreement over random networks. IEEE Trans. Autom. Control, 50:1867$72,2005$.

[27] R. A. Horn and C. R. Johnson. Matrix Analysis. Cambridge Univ. Press, 1985.

[28] H. Ishii and R. Tempo. Computing the PageRank variation for fragile web data. SICE J. Control, Measurement, and System Integration, 2:1-9, 2009.

[29] H. Ishii and R. Tempo. Distributed PageRank computation with link failures. In J. C. Willems et al., editor, Perspectives in Mathematical System Theory, Control, and Signal Processing, volume 398 of Lect. Notes Contr. Info. Sci., pages 139-150. Springer, Berlin, 2010.

[30] H. Ishii and R. Tempo. Distributed randomized algorithms for the PageRank computation. IEEE Trans. Autom. Control, 55:1987-2002, 2010.

[31] H. Ishii, R. Tempo, and E.-W. Bai. Distributed randomized PageRank algorithms based on web aggregation over unreliable channels. In Proc. 49th IEEE Conf. on Decision and Control, pages 6602-6607, 2010.

[32] H. Ishii, R. Tempo, E.-W. Bai, and F. Dabbene. Distributed randomized PageRank computation based on web aggregation. In Proc. 48th IEEE Conf. on Decision and Control and Chinese Control Conference, pages 3026-3031, 2009.

[33] S. Kamvar, T. Haveliwala, and G. Golub. Adaptive methods for the computation of PageRank. Linear Algebra Appl., 386:51-65, 2004.

[34] A. Kashyap, T. Başar, and R. Srikant. Quantized consensus. Automatica, 43:1192-1203, 2007.

[35] H. K. Khalil. Nonlinear Systems, 2nd edition. Prentice-Hall, Uppersaddle River, NJ, 1996.

[36] G. Kollias, E. Gallopoulos, and D. B. Szyld. Asynchronous iterative computations with web information retrieval structures: The PageRank case. In G. R. Joubert et al., editor, Parallel Computing: Current and Future Issues of High-End Computing, volume 33 of NIC Series, pages 309-316. John von NeumannInstitut for Computing, Julich, Germany, 2006. 
[37] A. N. Langville and C. D. Meyer. Google's PageRank and Beyond: The Science of Search Engine Rankings. Princeton Univ. Press, 2006.

[38] Y. Lin, X. Shi, and Y. Wei. On computing PageRank via lumping the Google matrix. J. Comp. Applied Math., 224:702-708, 2009.

[39] C. D. Meyer. Stochastic complementation, uncoupling Markov chains, and the theory of nearly reducible systems. SIAM Rev., 31:240-272, 1989.

[40] S. P. Meyn and R. L. Tweedie. Markov Chains and Stochastic Stability, 2nd edition. Cambridge University Press, 2009.

[41] A. V. Nazin and B. T. Polyak. Randomized algorithm to determine the eigenvector of a stochastic matrix with application to the PageRank problem. Automation and Remote Control, 72:342-352, 2011.

[42] A. Papoulis and S. U. Pillai. Probability, Random Variables and Stochastic Processes, 4th edition. McGraw Hill, New York, 2002.

[43] R. G. Phillips and P. V. Kokotovic. A singular perturbation approach to modeling and control of Markov chains. IEEE Trans. Autom. Control, 26:1087-1094, 1981.

[44] A. Tahbaz-Salehi and A. Jadbabaie. A necessary and sufficient condition for consensus over random networks. IEEE Trans. Autom. Control, 53:791-795, 2008.

[45] R. Tempo, G. Calafiore, and F. Dabbene. Randomized Algorithms for Analysis and Control of Uncertain Systems. Springer, London, 2005.

[46] R. Tempo and H. Ishii. Monte Carlo and Las Vegas randomized algorithms for systems and control: An introduction. European J. Control, 13:189-203, 2007.

[47] C. W. Wu. Synchronization and convergence of linear dynamics in random directed networks. IEEE Trans. Autom. Control, 51:1207-1210, 2006.

[48] G. G. Yin and Q. Zhang. Continuous-Time Markov Chains and Applications: A Singular Perturbation Approach. Springer, New York, 1998.

[49] Y. Zhu, S. Ye, and X. Li. Distributed PageRank computation based on iterative aggregationdisaggregation methods. In Proc. 14th ACM Conf. on Information and Knowledge Management, pages $578-585,2005$. 